\title{
Analogical Argument Schemes and Complex Argumentation
}

\author{
ANDRÉ JUTHE \\ Myrvägen 26 \\ 74732 Alunda, Sweden \\ Affiliation: University of Amsterdam, The Netherlands \\ andre.juthe@gmail.com
}

\begin{abstract}
This paper addresses several issues in argumentation theory. The over-arching goal is to discuss how a theory of analogical argument schemes fits the pragmadialectical theory of argument schemes and argument structures, and how one should properly reconstruct both single and complex argumentation by analogy. I also propose a unified model that explains how formal valid deductive argumentation relates to argument schemes in general and to analogical argument schemes in particular. The model suggests "scheme-specificvalidity" i.e. that there are contrasting species of validity for each type of argument scheme that derive from one generic conception of validity.
\end{abstract}

Résumé: Cet article traite de plusieurs questions dans la théorie de l'argumentation. L'objectif englobant est de décrire comment une théorie des schèmes d'arguments par analogie correspond à la théorie pragma-dialectique sur les schèmes et les structures d'arguments, et comment on devrait reconstruire correctement les arguments par analogie simples et complexes. Je propose également un modèle unifié qui explique comment l'argumentation formelle déductivement valable se rapporte aux schèmes d'argument en général et aux schèmes d'arguments analogiques en particulier. Le modèle suggère une «validité-spécifique-au-schème» : pour chaque type de schème d'argument dérivé d'une conception générique de validité il y a différentes espèces de validité.

Keywords: analogical argument schemes; argumentation by analogy; vertical determining relation; horizontal one-to-one correspondence; formal validity; material validity; pragmatic validity; argumentation structure; reconstruction; scheme-specific-validity

\section{Introduction}

The purpose of this paper is to show how an analysis of analogical argument schemes previously done by the author relates to the pragma-dialectical account of argument schemes and complex argumentation structures and show how one should correctly reconstruct single and complex analogical argumentation in that framework. Thus, the pragma-dialectical distinction 
of argument schemes versus argument structures, and so on, is an assumed framework. The goal is also to understand how analogical argument schemes could fit into a larger model of argumentation which covers both formally valid argument patterns - as traditionally understood - and the typology of argument schemes in the pragma-dialectical system.

In order to fully satisfy this goal in the broad sense that I want, I need to achieve four subgoals:

(1) Explain how analogical argument schemes achieve transfer of the acceptability of the "argument" to the standpoint according to my theory of analogical inference. This is done in section [2]. In this section I also discuss how single argument by analogy should be reconstructed and interpreted within a pragma-dialectical framework.

(2) Show how my theory of analogical inference configuration outlined in section [2] fits into a larger model of argumentation that coherently explains inference and validity and explains the connection between formal logically valid patterns (like modus ponens) and argument schemes that fit with the pragmadialectical system. In this section, the notions of formal and material inference as well as "material validity," "pragmatic validity" and "scheme-specific validity" are explained in terms of the larger model. In this section, it is also argued that analogical argument schemes cannot be reduced to any of the other argument schemes in the pragma-dialectical system. This is all done in section [3].

(3) Show how to correctly reconstruct complex argumentation structures that employ analogical arguments in a way that displays all the critical aspects essential to evaluation, and how the Amsterdam school of dialectical of argumentation structures relates to the north American conception of logical argumentation structures. This is done in section [4].

(4) Argue that while particular contexts of communicative activity types may activate certain stereotypical argumentative patterns, the intrinsic nature of the schemes themselves also tends to activate certain stereotypical argumentative patterns as well in the sense that schemes 
will activate certain complex argumentation structures. This is done in section [5].

The reader should have an understanding of pragma-dialectical theory and the terminology thereof in order to fully assimilate the discussion of this paper. ${ }^{1}$

\section{Analogical argument schemes}

In this section, I will give an account of my theory on how to understand the analogical argument scheme. For a more elaborate discussion about this theory of analogical inference, see (Juthe 2005; 2009) ${ }^{2}$. In subsection [2.1] the basic theory of analogical inference configuration will be outlined. In subsection [2.2] the theory will be applied to a concrete example of single argumentation by analogy so that the reader understand how the theory work in concrete cases of single analogical argumentation.

\subsection{The basic components of analogical argument schemes}

In this subsection, I will look at the basic elements that any argumentation by analogy will essentially have and the abstract pattern of that scheme.

In the pragma-dialectical framework an argumentation can be either single or complex and the idea is that a complex argumentation is always composed of a combination of single argumentations. Thus, any complex argumentation can be broken down into single arguments. Each single argument instantiates one argument scheme and consists of a standpoint, an explicit premise (labeled the "argument" that supports the standpoint and the "linking premise" (often unexpressed) that

\footnotetext{
${ }^{1}$ In this paper, the term argument is used in the ordinary sense as the whole constellation with premises and the conclusion, however if the expression is "argument" (with double quotes) then it refers to the technical pragmadialectical meaning corresponding to reason. In the pragma-dialectical system, what is called "argument" does not refer to all the propositions that make up an argumentation. An argumentation in pragma-dialectics consists of three propositions: the standpoint; that is to be defended by an "argument"; and the linking premise that entitles the "argument" to be a reason for the standpoint. Argumentation can either be single argumentation consisting of only one argument or complex argumentation which is composed of many arguments.

${ }^{2}$ My theory of analogical inference has been inspired by Steinhart (2001); Burbidge (1990) and Weitzenfeld (1984).
}

(C) André Juthe. Informal Logic, Vol. 35, No. 3 (2015), pp. 378-445. 
enables a unique way of conveying the acceptability from the explicit "argument" to the standpoint. The linking premise of an argument functions as a justification of the inference of the "arg ument" to the standpoint. Argument schemes are forms of argument that model uniform patterns of atomic reasoning.

That is, you cannot find smaller pieces of reasoning than the inference configuration of an argument scheme and they are therefore "atomic" in that sense. An argument scheme is the inference configuration that justifies and entitles (or warrants in Toulmin's words) the inference of the argumentation. The schemes are distinguished by the type of inference configuration that transfers the acceptability of the argument to the standpoint. In the pragma-dialectical typology you have three main types of argument schemes: the symptomatic, causal and analogical argument schemes. ${ }^{3}$

An argumentation by analogy (or analogical argumentation) is an argumentation that employs an analogy as a means to justify the inference. The Collins English Dictionary captures the basic intuition of analogical reasoning when it defines it as "a form of reasoning in which a similarity between two or more things is inferred from a known similarity between them in other respects" (1991: 53). The standard pragma-dialectical account of the analogical argument scheme is:

\section{1. $\mathrm{Y}$ is true of $\mathrm{X}$, [standpoint]}

because: $1.1 \mathrm{Y}$ is true of $\mathrm{Z}$, [argument]

and: 1.1' $\mathrm{Z}$ is comparable to $\mathrm{X}$. [linking premise]

However, the linking premise $1.1^{\prime}$ of this scheme cannot achieve a license of the transference of the acceptability of the "argument" to the standpoint unless comparability is more than mere similarity. Everything is similar to everything in some respect and dissimilar in some other respect, therefore you cannot infer anything from mere similarity as such. In order to justify an inference something more than mere similarity is needed. Analogical argumentation is a species of analogical reasoning and it should capture this basic intuition.

In my view, all analogies are composed of four components and two critical relations. The components are the Analogue $_{\mathrm{A}}$, which is the source from which a predicate is transferred to and concluded about the Target-Subject. The

\footnotetext{
${ }^{3}$ Some have argued that these schemes can be reduced into two "super schemes": argument from sign and argument from similarity, see Hitchcock and Wagemans, (2011).
}

(C) André Juthe. Informal Logic, Vol. 35, No. 3 (2015), pp. 378-445. 
Target-Subject $_{\mathrm{TS}}$ is that to which one assigns a new predicate; the Assigned-Predicate AP $_{\text {is }}$ is the new predicate that is transferred,

mutatis mutandis, to the Target-Subject, because the element $\in$ "determines" the Assigned-Predicate and because this element is comparable to a counterpart element $\in^{*}$ in the Analogue $\mathrm{A}_{\mathrm{A}}$. This essential constitution of my theory of argumentation by analogy would have the following scheme in the pragma-dialectical system of single argumentation:

1. The Target-Subject ${ }_{\mathrm{TS}}$ has the Assigned-Predicate AP*.

1.1 The element $\in$ of the Analogue Th $_{\mathrm{A}}$ is comparable with element $_{\in}$ of the Target-Subject TS $_{\text {S }}$

(1.1' The element of the Analogue A $_{\mathrm{A}}$ element determines the Analogue $_{\mathrm{A} \text { 's }}$ Assigned-Predicate $_{\mathrm{AP}}$.)

In some cases, the "argument" would express the comparability and the linking premise expresses the determining relation as in the scheme above. However, sometimes the reverse will hold (see later in this present section for examples) so that the "argument" expresses the vertical determining relation and the linking premise would express the analogous relation:

1. The Target-Subject ${ }_{\mathrm{TS}}$ has the Assigned-Predicate* ${ }_{\mathrm{AP}}$.

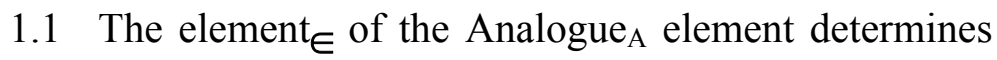
the Analogue $_{\mathrm{A} \text { 's }}$ Assigned-Predicate $_{\mathrm{AP}}$.

(1.1' The element $\in$ of the Analogue $_{\mathrm{A}}$ is comparable to element $_{\in}$ * of the Target-Subject ${ }_{\mathrm{TS}}$ )

The essential structure is, however, the same. Note that the argument scheme is formally invalid in the sense that the conclusion of the scheme does not follow by virtue of the logical concepts alone. Rather, the inference proceeds via a determining relation between the element $\in$ of the Analogue(A) and the Assigned-Predicate(AP) of the Analogue(A), which in logical symbols is not signified by logical entailment ' $\Rightarrow$ ' but is signified with the ${ }^{\prime}>-$ '. In this way, we define relevance. Thus the fact that $\in$ is relevant to AP is reduced to a determination (here I follow Davies 1988) written as $\mathrm{A}(\in)^{>-} \mathrm{AP}$. This "determination" is what I mean by the "vertical determining relation" and may be of any type; supervenience, resultance, causal, truthmaking, correlation, inferential, functional, genus to 
species, explanatory, etc., but can always be reduced to having two modes: definitely or non-definitely. Note furthermore that the determining relation says neither that the element $\in$ of the Analogue always determines the Assigned-Predicate nor that the element $\in$ of the Analogue in general determines the AssignedPredicate. Rather, it says that the element $\in$ determines the Assigned-Predicate in the Analogue. It is this particular Analogue's element $\in$ that determines this particular Analogue's Assigned-Predicate. This is important because it explains why my account of analogical inference cannot be reduced to any other type of inference configuration. The inference proceeds via an insight of resemblance between particulars and not via any general principle; the act of comparison is essential for the inference.

The inference is same-level-reasoning that moves from particular to particular (or from general to general) and you could not infer that a vertical determining relation exists in the Target-Subject unless you knew that it obtained in the Analogue and that there were corresponding elements in the TargetSubject. Thus, without the act of comparison and the similarity between the Analogue and the Target-Subject, you could not infer that the elements of the Target-Subject stand in the same vertical determining relation. This also explains why analogical inference is defeasible in the traditional sense and "valid" in the sense defined in this paper - a validity that allows the inference to be defeated (see section [3.3] for more about my account of "validity"). It explains why there is always an issue concerning relevant similarities and relevant differences concerning argumentation by analogy.

The comparability operates as the justification of the inference by means of which the same type of vertical determining relation can be inferred to hold in the TargetSubject. 'Comparability' means that each element in the Analogue that has a vertical determining relation to the Assigned-Predicate also has a horizontal one-to-one correspondence to a counterpart element in the Target-Subject. Thus, the transference of the Assigned-Predicate is justified because the element $\in$ in the Analogue corresponds one-to-one to a counterpart element $\in^{*}$ in the Target-Subject. Thus, the terms 'resemblance', 'similarity', 'comparable' or 'analogous' in the argument mean:

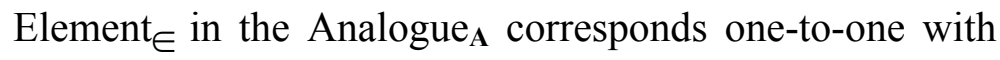
element $_{\in} *$ in the Target-Subject ${ }_{\mathrm{TS}}$. 
Thus, any argumentation by analogy tacitly assumes that the element(s) of the Analogue are comparable to the element(s) in the Target-Subject, and that the Assigned-Predicate mutatis mutandis (Assigned-Predicate*) therefore can be transferred from the Analogue onto the Target-Subject by means of the determining relation that can be extended to hold in the TargetSubject by virtue of the comparability.

As stated, the sense of 'determining' is broad and, as such, only indicates that there is some kind of material connection between the determining element and the Assigned-Predicate in the Analogue so that the connection can be transferred via the one-to-one correspondence. Thus, analogical inference is an example of materially valid inference in virtue of a substantial argument scheme. An argumentation that employs a correct analogy is materially valid, although not formally valid and, if it is consistent with the rules of the critical discussion, it will be pragmatical valid as well. (These concepts are all explained in section [3.3].) While you could supply the "logical minimum", that would make an analogical argumentation formally valid:

If the Analogue's element $\in_{1}, \in_{2} \ldots \in_{\mathrm{n}}$ determines Assigned-Predicate in the Analogue, and the TargetSubject also has element $\in_{1}, \in_{2} \ldots \in_{\mathrm{n}}$ (or a mutatis mutandis corresponding element $\in_{1} *, \in_{2} * \in_{\mathrm{n}} *$ ), then, unless there is another element in the Target-Subject that counteracts the effect of element $\in_{1}{ }^{*}, \in_{2}{ }^{*} \in_{\mathrm{n}}{ }^{*}$, the Target-Subject's element will also determine the Assigned-Predicate (or a corresponding mutatis mutandis Assigned-Predicate*) in the Target-Subject)

- the same can be done with any argumentation including every inductive argumentation ever made. Such reformulations have no positive merit, but are de facto negative, in that they confuse necessary background assumptions with unexpressed linking premises and make the argument into a non-analogical deductive modus ponens that only preserves truth and not plausibility. (See section [3] for more about this point.) A genuine argumentation by analogy is an argumentation that

\footnotetext{
${ }^{4}$ The logical minimum is the minimum proposition that states explicitly what makes the reasoning in the argumentation formally valid without contributing anything new. For instance if the argument is: "Steve must be at home, because his car is at home" then the logical minimum would be "If Steve's car is at home then Steve must be at home."
}

(C) André Juthe. Informal Logic, Vol. 35, No. 3 (2015), pp. 378-445. 
employs an analogy to justify the inference of the argumentation. If each element in the analogue that vertically determines the Assigned-Predicate has a counterpart in the Target-Subject so that each has a one-to-one correspondence, then the vertical relation in the analogue can be extended and conveyed to the Target-Subject and the Assigned-Predicate* of the corresponding domain can be concluded about the target subject. Just having a deductive modus ponens with a premise that "contains an analogy" is not an analogical inference.

Moreover, I believe that the concept of "relative essence" is another crucial concept of analogical inference where the elements that stand in a one-to-one correspondence constitute

\footnotetext{
${ }^{5}$ You would not claim that a deductive argument with empirical premises is an "inductive argument." Besides being clearly counter-intuitive, a taxonomy in which argument types are defined by the content and not by its inference configuration entails an infinite number of argument types. During a seminar in which the manuscript of this paper was under discussion, one of the participants of the seminar suggested an alternative analogical argument scheme in an attempt to avoid the vertical determining relation. According to the suggestion the argument scheme could be this:

(1) $\mathrm{x}$ and $\mathrm{y}$ both have A;

(2) Two things that have A are also similar with respect to $B$ (that is either both have B or both lack B);

(3) $\mathrm{x}$ has B;

(4) Thus y has B.
}

However this does not attain any analogical inference. Instead it is just a deductive argumentation, with a superfluous premise. It is either modus ponens or the disjunctive syllogism. The argumentation can either be interpreted as:

(1) $\mathrm{x}$ is similar to $\mathrm{y}$ with respect to $\mathrm{A}$;

(2) If something is similar with respect to $\mathrm{A}$, then it is also similar with respect to $\mathrm{B}$;

(3) $\mathrm{x}$ has B.

(4) y has B.

This makes the argument an instance of the formally valid modus ponens with (3) being superfluous. It can also be interpreted as:

(1) $\mathrm{A}(\mathrm{x}) \& \mathrm{~A}(\mathrm{y})(\mathrm{x}$ and $\mathrm{y}$ have $\mathrm{A})$

(2) $\mathrm{B}(\mathrm{x}) \& \mathrm{~B}(\mathrm{y}) \vee \sim \mathrm{B}(\mathrm{x}) \& \sim \mathrm{B}(\mathrm{y}) \quad(' \vee$ ' is here interpreted as exclusive). (Either $\mathrm{x}$ and $\mathrm{y}$ both have $\mathrm{B}$, or neither of them has $\mathrm{B}$ )

(3) $\mathrm{B}(\mathrm{x})$.

(4) $\mathrm{B}(\mathrm{y})$.

This is an instance of the disjunctive syllogism with again (3) being redundant. Hence it is not an analogical inference, it is a deductive inference.

(C) André Juthe. Informal Logic, Vol. 35, No. 3 (2015), pp. 378-445. 
what is "essential" to a certain framework of context. Two objects being analogous means that they have the same essential features, but 'essential' means only essential with respect to a certain contextual framework. This notion cannot be explicated here but is further elaborated in (Juthe forthcoming b).

\subsection{An example of single argumentation by analogy}

In this subsection, I will apply my explication of the analogical argument scheme to a concrete example of single argumentation by analogy. We will take a closer look at the art of reconstructing complex analogical argumentation in section [4], and will here only focus on the reconstruction of single argumentation. The example is a bit ambiguous with respect to how it should be interpreted, which I think will clarify for the reader how my theory of analogical argument schemes should be applied. The single argumentation:

The Porsche and the Chevrolet are both in the $\$ 40,000$ price range, and the Porsche is of excellent quality. Therefore, the Chevrolet is probably also of excellent quality. ${ }^{6}$

has the determining vertical relation between the "Porsche's $\$ 40,000$ price range" and the "Porsche's excellent quality." Thus, the element " $\$ 40,000$ price range," in the case of the Porsche either definitely or non-definitely determines its "excellent quality." Thus, the reconstruction with all tacit assumptions is:

1. The Chevrolet TS $_{\mathrm{T}}$ is probably of excellent quality . $_{\mathrm{AP}}$

1.1 The $\$ 40,000$ price range $\in$ of the Porsche $_{\mathrm{A}}$ corresponds one to-one with the price range of the Chevrolet $_{\in} *$.

$1.1^{\prime}$ The $\$ 40,000$ price range $_{\in}$ of The Porsche $_{\mathrm{A}}$ nondefinitely determines excellent quality $\mathrm{AP}$.

For sake of convenience, I will continue to use the terms

\footnotetext{
${ }^{6}$ In a previous work (Juthe 2005) I claimed that this argument was a distinct type of "argument by similarity" and could not be considered as an argument by analogy. I have, however, changed my conviction, in this respect, since I think that even the most simple argument by analogy which compares just one property with another property in the Target-Subject also has a vertical relation even though it may be completely unexpressed.
}

(C) André Juthe. Informal Logic, Vol. 35, No. 3 (2015), pp. 378-445. 
'relevance' and 'comparable' or 'analogous' but in the sense defined in the previous subsection. Thus, the above reconstruction will be simplified as:

1. The Chevrolet is comparable with the Porsche with respect to excellent quality.

1.1 The Chevrolet is comparable with the Porsche with respect to $\$ 40,000$ price range.

(1.1' The $\$ 40,000$ price range of the Porsche is relevant for its excellent quality.)

I want to emphasize that there is more than one proper way to reconstruct the same analogical argumentation, as long as the reconstruction retains the crucial features relevant for an evaluation in a given situation. However, although there may be more than one proper way to reconstruct analogical argumentation there are far more erroneous ways to do it. Sometimes it is very tricky to settle how to reconstruct an argumentation. For instance, one could claim that this argumentation should also be reconstructed as: ${ }^{7}$

1. The Chevrolet is probably of excellent quality.

1.1 Porsche is of excellent quality.

(1.1' The Chevrolet is comparable with the Porsche)

$1.1 ' .1$ The Chevrolet is also in the $\$ 40,000$ price range.

This is also a prima facie good reconstruction. Notice that this latter reconstruction interprets the argument as complex (with one subordinative addition). The question is, should "Both are in the $\$ 40,000$ price range" be taken as the result of a dialectical move, or as something-part of the intrinsic structure of the scheme itself - that resides in any "valid" analogical argument?

My assessment is that this argumentation should be interpreted as single argumentation. Note that the linking premise is imprecise in this formulation and if we make it more precise we will see difficulties. If we specify it as: "The Chevrolet is comparable with the Porsche with respect to quality," it would be circular reasoning, since that is what the argument is supposed to "prove" (and such an interpretation would violate the principle of charity).

However, if we specify it as: "The Chevrolet is comparable with the Porsche with respect to price," then the argument becomes different, because it no longer says the same

\footnotetext{
${ }^{7}$ This reconstruction was suggested by Bart Garssen.
} 
thing. It is a very different claim to claim that: "its $\$ 40,000$ price range is relevant to the excellent quality of the Porsche" and to claim that: "price range in general is relevant for quality in general." The latter claim makes it into a symptomatic argumentation, since you do not need knowledge about the particular Porsche any longer. It makes the knowledge about the Porsche redundant. You can use that generalization alone to justify the inference. Such a formulation would also need an additional linking proposition that expresses that price range is relevant for quality:

1. The Chevrolet is probably of excellent quality.

1.1 The Porsche is of excellent quality.

(1.1' The Chevrolet is comparable to the Porsche with respect to price range.

(1.1" Price range is relevant to quality.

$1.1^{\prime} .1$ The Chevrolet is also in the $\$ 40,000$ price range.

This seems to stretch the argumentation into a more complex reconstruction than necessary. There is no evidence for the claim that the pre-reconstructed argumentation assumes 1.1". It violates the criterion for establishing the committed premise (see section [4.2]) since it assumes more than what is necessary to make the argument materially valid (see next section for an explanation of this concept).

Moreover, this reconstruction would violate the pragmadialectical axiom that each single argumentation consists of two propositions, the "argument" and the "linking premise," support the standpoint. Therefore, there are reasons to interpret the argumentation as single without any dialectical addition. The logic of this analogical inference can be seen visually in Figure 1 , on the next page.

The non-definitely determining relation of the vertical relation makes the argument an "argument by inconclusive analogy" in contrast to analogical arguments that have a definite vertical determining relation, which makes the argument an "argument by conclusive analogy." (This distinction is more elaborated in Juthe 2005; 2009.)

\footnotetext{
${ }^{8}$ Nor yet can you make 1.1' and 1.1" missing parenthesis into just one linking premise by expressing them as one conjunction because that would be inconsistent with how you in general express the connection between the argument and the linking premise in the pragma-dialectical system. 1.1 and $1.1^{\prime}$ is supposed to be one single argumentation in itself and 1.1' 1. another (with its own unexpressed linking premise) thus the 1.1" is an anomaly if we interpret the argumentation as complex argumentation.
}

(C) André Juthe. Informal Logic, Vol. 35, No. 3 (2015), pp. 378-445. 


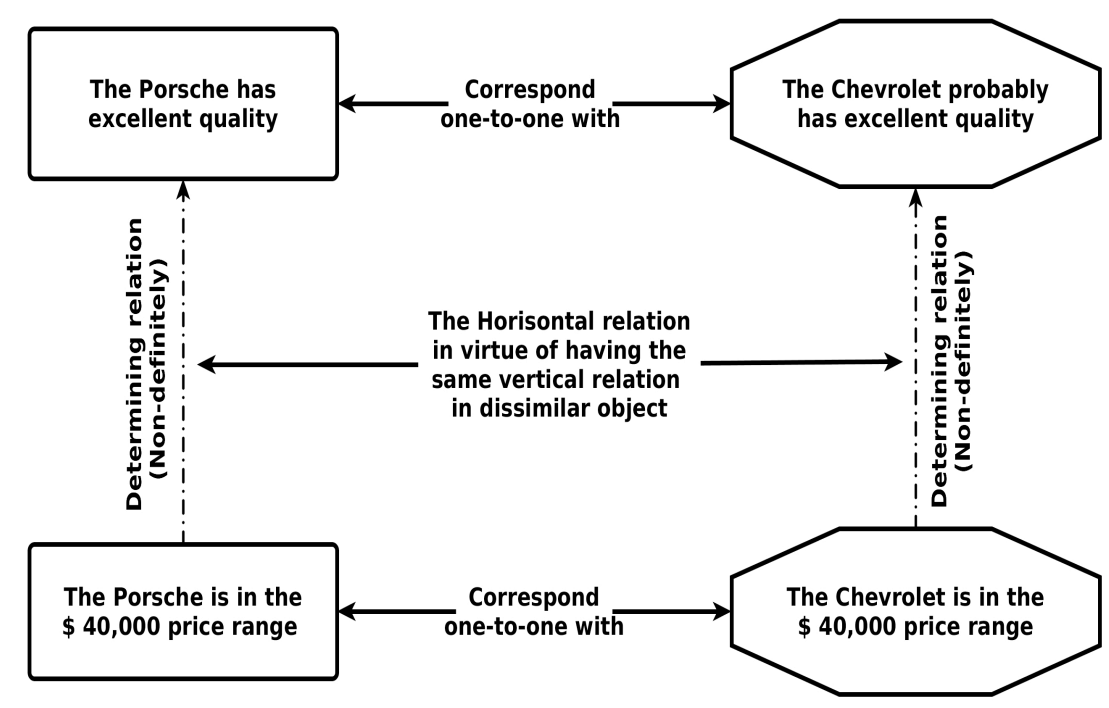

Figure 1. Single argumentation by inconclusive analogy

While a price range surely correlates with quality, it does not definitely determine quality. The determination relation between price and quality is plausibly never stronger than a correlation, which is the reason why the standpoint has the mode of "probably." The fact that the Target-Subject has a counterpart element for each of the analogue's element entails that there is a "horizontal relation" as well, which enables the analogical inference.

It is not my claim that an analogical argument has to be explicit in exactly which features are relevant for the comparison. You do not necessarily need to know exactly which they are in order to make a correct comparison. The elements and the vertical determining relation may often be tacit or unknown, which entails that you neither can account for the oneto-one correspondence.

A comparison is often just based on an intuitive judgment of similarity, as Govier points out: "The trick about analogiesand their charm as well, I think - is that we are often able to see or sense important resemblances between cases without being able to spell them out exhaustively in just so many words" (Govier 1989, p. 148). ${ }^{9}$ The point is rather that, according to my

\footnotetext{
${ }^{9}$ Govier seems to claim that if we knew which similarities it was we could transform the argument into a deductive one, and here I disagree with her, since knowing certain similarities is insufficient for making a universal or
}

(C) André Juthe. Informal Logic, Vol. 35, No. 3 (2015), pp. 378-445. 
theory of analogical inference, all genuine analogical arguments are committed to the existence of these components even though they may be completely unknown and implicit, just like a deductive argument may be committed to several implicit assumptions that we are not aware of, although we still understand that the argument is formally valid without being able to formulate the unexpressed linking premise. For example, the very simple argumentation:

This vaccine works on cows. Therefore it may also work on humans.

would be an example of advancing a single argumentation by analogy and the standard scheme in pragma-dialectics would reconstruct it ${ }^{10}$ :

1. The vaccine may work on humans. [Standpoint]

1.1 The vaccine works on cows. [Argument]

(1.1' Cows are comparable to humans.) [Implicit linking premise]

As we saw in the previous subsection, my theory of analogy entails that every single argument that employs an analogical argument scheme contains the same two crucial assumptions. Thus, the linking premise $1.1^{\prime}$ is an elliptic expression of the proposition that de facto expresses the horizontal one-to-one relation between the Target-Subject and the Analogue. The second assumption of the vertical determining relation applied to this particular single argument, would be that a "basic physiological feature $\in$ " causally but non-definitely determines the positive effect of the vaccine on cows. Since it has a counterpart "basic physiological feature $\in$ *" of humans, so that the Assigned-Predicate, the "positive effect of the vaccine," can be transferred to the human population. Thus it should be reconstructed:

1. The vaccine may work $\mathrm{AP}_{\mathrm{AP}}$ on humans ${ }_{\mathrm{TS}}$ [Standpoint] 1.1 The vaccine works $\mathrm{AP}$ on cows $_{\mathrm{A}}$ because of some

even general claim, since there may be dissimilarities that counteract and outweigh the similarities. See also Guarini (2004) pp. 156, 163.

10 This argument might be interpreted as one employing a symptomatic argument scheme as well as an analogical one; however, for the sake of the argument, we will assume the latter.

(C) André Juthe. Informal Logic, Vol. 35, No. 3 (2015), pp. 378-445. 
basic physiological feature $\in$ of $\operatorname{cows}_{\mathrm{A}}{ }^{11}[$ argument that express vertical determining relation]

(1.1'. The basic biological feature ${ }_{\in}$ of Cows $_{A}$ is analogous to the basic biological feature $\in^{*}$ of humans TS.) [linking premise that express the horizontal one-toone relation]

The term comparable or analogous in single arguments should be analyzed in terms of the components discussed earlier. Therefore, my theory assumes that the linking premise 1.1' means:

basic physiological feature $\in$ of cows corresponds one-toone with basic physiological feature $\in^{*}$ of humans.

This does not mean that the author of the argumentation is committed to the claim that he knows what these basic physiological features are (they may be totally unknown); the point is solely that the author of this argument is committed to the ontological claim that they exist.

The "because" of this determining relation in the case of how the vaccine works on cows is loose, and hence it is an example of argument by "inconclusive analogy." Presumably, the vaccine did not work on every cow and therefore only a correspondingly probabilistically qualified predicate can be transferred to the Target-Subject in the standpoint-in this case humans - in the analogical inference. Just as a correct inductive inference does not yield a certain conclusion, only presumptive or probabilistic, arguments by inconclusive analogy only yield a plausible conclusion regardless of how certain the analogy may be.

Thus, my theory assumes that argumentation like this very simple one is committed to the implicit assumption that the similarity in question is relevant for (determines) the effect of the vaccine, and that not every similarity is relevant, even though it is not specified exactly what these relevant similarities consist of. However, most cases of argumentation by analogy provides information about what elements are part of the determining relation and how they are related.

Besides the distinction between argument by "inconclusive" analogy and argument by "conclusive" analogy, there is

\footnotetext{
${ }^{11}$ In other words, the basic physiological feature $\in$ is relevant for the vaccine working on cows.
}

(C) André Juthe. Informal Logic, Vol. 35, No. 3 (2015), pp. 378-445. 
a distinction between argument by same-domain and differentdomain analogy (Juthe 2005; 2009; 2014) as well as closedomain analogy (Juthe forthcoming b).

A same-domain analogy means that the AssignedPredicate does not change in the analogical inference when transferred to the Target-Subject, because they belong to the same domain, whereas in a different-domain and close-domain analogy, the Assigned-Predicate has changed mutatis mutandis in accordance with the domain of the Target-Subject so that a higher-level of abstraction is needed in order to subsume them under the same topical predicate.

Moreover, in a different domain-analogy, the elements in the one-to-one correspondence would consist of different predicates, whereas the one-to-one corresponding elements in a same-domain analogy would consist of the same type of predicate (even though the particular objects of comparison are not the same).

This example with the cars, is a same-domain analogy because: (1) the object of comparisons belong to the same domain: the domain of cars, and the Assigned-Predicate does not change mutatis mutandis at all, and (2) the predicate-element " $\$ 40,000$ price range" is the exactly the same in both the Analogue and in the Target-Subject (in different-domain analogies the predicate-element would be dissimilar in accordance with their respective domains).

\section{The "validity" and the sui generis character of analogical inference}

I have in section [2] explained how the inference operates in analogical argument schemes and how to reconstruct single analogical argumentation. In this section [3], I will give an account of the "validity," and defend the sui generis character of the analogical inference that was outlined in previous section [2], and explain how analogical argument schemes fit into a unified model of inference and the "validity" of argument schemes. I will argue that we should reject the traditional notion of validity as a concept that only means the formal validity of deductive arguments. Instead, I argue that every instance of validity should be taken as a differentiated species of a generic conception. The differentiation depends on which type of argument scheme an argumentation employs and each type of scheme has its own specific standard of validity.

(C) André Juthe. Informal Logic, Vol. 35, No. 3 (2015), pp. 378-445. 
In subsection [3.2], I will define and explain how I understand 'inference' and 'material inference.' In subsection [3.3], we will take a closer look at the crucial notions of the material and pragmatic validity of analogical inference and contrast it with "formal validity" and how these notions fit into a unified model. In subsection [3.4], I introduce the notion of scheme-specific validity, which means that validity is specific for each type of argument scheme. Scheme-specific validity follows from the unified model of argument schemes.

Finally, in subsection [3.5], I argue that analogical inference is sui generis and cannot be reduced to some other type. I have elsewhere argued that arguments by analogy cannot be reduced to deductive types of argument (Juthe 2005, 2009) and many other theorists have argued for that as well (Govier 1989; Guarini 2004; Bermejo-Luque 2014).

However, that argument by analogy cannot be a genuine inference configuration by itself and must be reduced to some other type of inference is a common view (see Shecaira 2013 for a recent defense of such a view and for deductivism). I will therefore in this section provide further support that the schemes of argument by analogy are indeed legitimate argument schemes; but I will focus on the question whether the analogical argument scheme can be reduced to a symptomatic or causal argument scheme. Before all this, however, we will in the first subsection [3.1] take a brief look at deductivism.

\subsection{Deductivism}

Deductivism may roughly be described as the view that all arguments should be interpreted and/or evaluated as attempts to employ deductive argumentation, or that all good arguments are deductively valid, i.e., formally valid (exactly how I define 'validity' will be discussed in section [3.3]). There are many problems with deductivism (Godden 2005) ${ }^{12}$, and one reason against deductivism is that arguments with deductive architecture only preserve truth and certainty, they fail to preserve plausibility, probability and likelihood (Godden 2005). ${ }^{13}$ Another problem are those arguments that are

\footnotetext{
${ }^{12}$ Several of them are advanced by Godden (2005) and refers to Govier. The standard objections are: (i) deductivism prevents contrasting degrees of evidential support between premises and conclusions; (ii) deductivism fails to provide an account of fallacies, or the account it provides is a faulty account of fallacies; (iii) deductivism does not do justice to the structure of natural language arguments.

${ }^{13}$ Godden (2005) provides several examples. Godden also argues that they
}

(C) André Juthe. Informal Logic, Vol. 35, No. 3 (2015), pp. 378-445. 
seemingly counterexamples in that they resist a plausible deductive formulation and are better arguments in their nondeductive formulation. An example of what some authors call inductive analogical argument: ${ }^{14}$

(1) $\quad a, b, c \ldots$ each has been observed to have property $F$ and property $G$;

(2) $n$ is observed to have property $F$;

Therefore, probably $n$ has property $G$

The very same argument should according to a deductivist interpretation be formulated with a deductive step, making it formally valid:

(1*) $a, b, c \ldots$ each has been observed to have property $F$ and property $G$;

$\left(2^{*}\right) n$ is observed to have property $F$;

(3*) All $F$ s are $G^{\prime}$ s

$\overline{\text { *Therefore } n}$ is $G$ (or: $\mathrm{n}$ is probably $G$ )

However, this is an essentially different argumentation, since the method and outcome of the evaluation of this reformulated argumentation would be completely dissimilar from the first argument. The first argument works via induction and resemblance, whereas the other via a universal generalization. What is interesting here is that the first inductive version is, from an epistemic point of view, a clearly better argument than the deductive version, since it is not based on the very questionable premise (3). ${ }^{15}$ As Barker pointed out, we are not entitled to attribute higher likelihood to the conclusion of the deductive version, than the likelihood we are entitled to assign to the generalization about $A l l F^{\prime}$ s are $G^{\prime} s$. Yet we do frequently reach particular conclusions with a considerably higher degree of plausibility than we are in making such generalizations (Barker 1989, makes this point, pp. 173-177, see especially p.175). Thus, such reasoning goes more plausibly from case to case and not via a universal generalization and/or by virtue of

fail to "properly represent the nature of inference warrants as distinct from premises" (p. 174).

${ }^{14}$ Barker S.F. (1989) calls it an "inductive argument by analogy".

${ }^{15}$ Others, like Reidhav has made the same points see Reidhav (2007, pp. 3237). 
logical form. As Bertrand Russell noted:

The probability of the general law is obviously less than the probability of the particular case, since if the general law is true, the particular case must also be true, whereas the particular case may be true without the general law being true. (Russell [1913] 2013, chapter VI)

Thus, dissimilar formulations of one and the same argument will yield dissimilar argumentative merits, a result that should be unacceptable and is evidence that such deductive reformulation yields essentially different arguments. Although you could always employ the qualifier "probably" and reformulate an essentially inductive argument into a deductive, the question remains how this would make the argument better from any point of view. In a trivial sense you can always add the "if..., then" clause to any set of propositions asserting if these propositions, then this proposition, and therewith making it into a formally valid argument.

But such a move would be quite meaningless if you are interested in correctly reconstructing an argumentation in order to evaluate its argumentative merits. It says nothing about how or by which means the premises are related to the conclusion with respect to other aspects than just logical form, even something that may be crucial when you want to evaluate the argumentation. Reformulating an essentially non-deductive argument into a deductive one, transforms the question of evaluation the inference link into a question of the truth of the premises. Is there any reason to believe that such transformation has any real value?

\subsection{Material inference and substantial argument schemes}

In this section, I will define 'formal argument schemes' versus 'substantial argument schemes' and 'formal inference' versus 'material inference.'

An "inference" is the transference of the acceptability of an "argument" to a "standpoint" by means of a linking premise in an argumentation scheme. An "argument scheme" is a fixed pattern of inference configuration that enables a proposition labeled "argument" to give reason for another proposition, "the standpoint" by virtue of another proposition-the "linking premise"- that operates in conjunction with the "argument." The scheme is the "syntax" of an argumentation. The inference configuration of an argumentation scheme is "atomic" in the 
sense that it cannot be broken down into fewer components and retain any inference configuration.

A "single argumentation" is intended to be one concrete instance of one argument scheme. While all inference is based on the "syntax" of an argument scheme and the meaning of the concepts therein, it can be subdivided according to whether the inference occurs via logical concepts alone or whether factual concepts play a role as well; whether the argument scheme has a definite material content as part of its syntax. All inference is based on the meaning of the concepts of the argument scheme, but there is a contrast between the types of concepts, logical or factual, that justify the inference. I will here explicate the notion of material inference (Bringandt 2010). The idea of "material inference" is to some extent inspired by Bringandt (2010), but also a development of some ideas expressed earlier (Juthe 2005, 2009).

Although all inferences are material in the sense that no concept is never completely devoid of material content, I do distinguish between formal (or logical) and substantial (or material) argument schemes.

I see no reason for the claim that patterns like modus ponens, and modus tollens, etc., are not "argument schemes" if we hold to the definition just outlined. Logical patterns like modus ponens and the syllogisms, are also fixed patterns of "inference configurations" that enable one proposition (the "minor premise") to give reason for another proposition, the "conclusion," by virtue of another proposition (the "major premise"') that operates in conjunction with the "minor premise."

This general description holds both for argument schemes and the logical forms that are sometimes labeled "reasoning schemes." The formal inference configuration shares everything that is essential to the general inference configuration of argument schemes traditionally understood. Such a perspective provides a unified model of the taxonomy of argumentation where argumentation that employs formal reasoning is just another type of argumentation. ${ }^{17}$ You cannot object that my model confuses different levels of an argumentation (i.e., that also substantive argument schemes must instantiate patterns as

\footnotetext{
${ }^{16}$ Immediate deduction is of course exception to this general but not universal description.

${ }^{17}$ This does not exclude that you make a distinction when you employ the pattern in kinds of reasoning types other than argumentation, like for example, explanatory reasoning.
}

(C) André Juthe. Informal Logic, Vol. 35, No. 3 (2015), pp. 378-445. 
modus ponens, or modus tollens). ${ }^{18}$ The internal "logical" structure of arguments by analogy is the scheme that was outlined in section [2] and even if these substantial schemes in turn presuppose the logical minimum stated in section [2.1] and/or perhaps the proposition: "if, this scheme, then this conclusion" as an assumption, such assumptions are not unexpressed premises but rather necessary background assumptions, and this also holds equally for formally valid "argument schemes." Thus, the "logical minimum" should not be thought of as a gap-filling premise in substantial argument schemes but as a background assumption. In fact every argument scheme of any type as well as any formal valid scheme assumes a benign infinite regress of such "logical minimum's.",19

Moreover, there is another route you can take. You could deny that an author of argumentation that employs a substantial argument scheme is committed to the logical minimum as presupposition, and claim that the author is rather committed to claims like: "my arguments are good grounds for the standpoint," or "these reasons are good ones," etc., (Godden 2005, makes this point p. 179).

The idea that every argumentation besides instantiating an argument scheme also must have a formally valid logical form, like modus ponens or modus tollens as inference configuration in order to transfer acceptability the standpoint, is incoherent. It would make any talk about causal or symptomatic or analogical argument schemes pointless. The inference would work by virtue of logical form alone and not by any substantial principle. $^{20}$ It would reduce the testing procedure of the inference to solely a question of the truth of the premises. Rather, the logical form is a necessary condition for the inference in argument with a substantial argument scheme

18 You cannot claim that it is only a matter of how "externalized" the argumentation is; that would beg the question as to why we should assume that every externalized argumentation should be formally valid. The question is also how you can be justified to assign an argument scheme to an argumentation that makes the reconstruction formally invalid, while you simultaneously claim that the original pre-reconstructed argumentation in reality is (when fully externalized) formally valid.

19 That is, if we accept that substantial argument schemes like the symptomatic and causal and analogical scheme assume such a presupposition, then so does any of the "reasoning schemes" as well.

${ }^{20}$ Robert Pinto makes another classification by distinguishing between truthpreserving rules/patterns and entitlement-preserving rules/patterns, see Pinto (2006). However his model cannot provide a unified model of inference and validity.

(C) André Juthe. Informal Logic, Vol. 35, No. 3 (2015), pp. 378-445. 
whereas it is a necessary and sufficient condition for the inference of an argument employing a formal argument scheme. This model fits well with the revision of the ten rules of the critical discussion in the pragma-dialectical system (van Eemeren \& Grootendorst 2004, pp. 193-195). ${ }^{21}$

In my view, substantial argument schemes of course have a "logical form," but their internal structure needs a particular material content in order to function as an inference configuration. The inference does not proceed via a universal generalization or in virtue of the logical form alone. Therefore, my point is that while every argumentation does have a logical structure in some sense, only a deductively valid argumentation can be reduced to an inference configuration that is formally valid, i.e., that transfers acceptability by virtue of logical concepts without any reference to material content. Thus, while a substantial argument at the logical level does have a (logical) structure, it cannot operate on that level alone in order to transfer acceptability to the standpoint. Substantial argument schemes have a logical structure as a necessary presupposition,

${ }^{21}$ In van Eemeren et al. (2002) you have the rule 7, the Argument scheme rule: "A standpoint may not be regarded as conclusively defended if the defense does not take place by means of an appropriate argument scheme that is correctly applied." (p. 130), and rule 8, the Validity rule: "The reasoning in the argumentation must be logically valid or must be capable of being made valid by making explicit one ore more unexpressed premises." (p. 132). With this formulation there is a discordance between rule 7 (Argument scheme rule) and rule 8 (the validity rule) of the ten rules of the critical discussion. If every reasoning in an argumentation must be formally valid, there would be no need for argument schemes (if traditionally understood). However, in van Eemeren \& Grootendorst 2004 pp. 193-195) the rules are reversed and revised so that rule 7 is the validity rule which now states: "Reasoning that in an argumentation is presented as formally conclusive may not be invalid in a logical sense." and rule 8 is the argument scheme rule: "Standpoints may not be regarded as conclusively defended by argumentation that is not presented as based on formally conclusive reasoning if the defense does not take place by means of appropriate argument schemes that are applied correctly." In this revision there is a crucial difference between argumentation that presents itself as a "formally conclusive" argumentation that according to such standards must be formally valid, whereas argumentation that is not presented as based on formally conclusive reasoning, must employ an appropriate argument scheme that has its own standards as to when the inference is entitled. Thus, rule 7 and rule 8 refer to contrasting standards for two separate groups of arguments that are contrasted according to the means by which they authorize their inference. This fits well with the model I am suggesting in this paper. While non-formal arguments may require a logical structure as a necessary presupposition, they do not employ it as the efficient principle that operates in the inference as a linking premise in contrast to formally valid arguments.

(C) André Juthe. Informal Logic, Vol. 35, No. 3 (2015), pp. 378-445. 
but do not employ any proposition that makes the argument formally valid (such as the logical minimum or something similar) as the linking premise, in contrast to formally valid argument schemes. ${ }^{22}$ The linking premise expresses the efficient principle that operates to entitle the inference in an argument, and the linking premise of substantial argument schemes always have a material content as a necessary component. ${ }^{23}$

A formal argument scheme makes the "argument" a relevant reason for the standpoint, ceteris paribus, by means of the logical concepts in the scheme alone, whereas a substantial argument scheme (like the symptomatic, causal or analogical) makes the "argument" a relevant reason for the standpoint, ceteris paribus by means of the substantial content of the linking premise, and not the logical concepts alone. While there is a generic material inference there is distinction between schemes that employ a "formal inference" and those that employ a "material inference." A correct formal scheme yields a formal inference and a formally valid argumentation, whereas a correct material scheme yields a material inference and a materially valid argumentation.

However, although I make a distinction between formal and substantial argument schemes, I reject the notion of empty "pure laws of form" completely separated from matter. All inferences are ultimately based on a material aspect since all inferences are based on how the meaning of our concepts succeeds in mirroring reality and logical concepts are no different in that respect. That means that you cannot assume the universal validity of even the modus ponens, etc.

All inferences, even formal ones, are to some extent, material in their nature. The distinction between inference by "logical concepts" and "factual concepts" is simply not

\footnotetext{
${ }^{22}$ For instance, an argument instantiating " $Y$ is true of $X$, because $Z$ is true of $X$, and $Z$ is typical/symptomatic of $Y$ " might presuppose the universal: "If $Z$ is typical/symptomatic of $Y$, then probably $Y$ is true of $X$." However, as I have emphasized again and again, such a proposition would be an implicit background assumption and not a gap-filling linking premise.

23 This seems to be consistent with what other theorist in the pragmadialectical schools have held. Bart Garssen (2001) states (p. 81), that "Each argument scheme represents a specific principle of support.", and in the footnote of this sentence (p. 96) he states: "Argument schemes are general and abstract patterns with an infinite number of possible substitution instances. In this respect they correspond to logical reasoning patterns. With an argument scheme, however, the transfer of acceptability is based on more than just the formal characteristics of the scheme that is used" (emphasis added). Plausibly, this 'more' is the specific material principle that enables the transference of acceptability to take place in "substantial schemes."
}

(C) André Juthe. Informal Logic, Vol. 35, No. 3 (2015), pp. 378-445. 
absolute, just as the distinction between "analytical" and "synthetic" truths is not an absolute one (as Quine has argued). Thus, while I make the distinction between formal and substantial argument schemes by virtue of contrasting sources of the justification of the inferences (the meaning of logical concepts vs. the meaning of non-logical factual concepts), I simultaneously maintain that the meaning of logical concepts is never completely empty of material content; they are essentially connected to the non-logical content they logically connect. Logic is both in the mind and in the world in a substantive sense, a sense that would not be possible unless the mind and reality both were dimensions that were necessarily interconnected.

The idea that there exist any pure abstract logical forms devoid of any factual content has epistemological, metaphysical and semantical objections and is even refuted by several counterexamples. Inference is always a subject-matter relationship. See for example: Theron (1997), Smith (1986, pp. 173-194; 1988, pp. 124-176), McGee (1985).

Besides these strong objections from semantics and counterexamples, we have two reasons provided by Brigandt (2010). The first is that formal inference can be analyzed in term of material inference but not vice versa. The class of formally valid inferences can be interpreted as the class of inferences that remain materially valid under any replacement of non-logical concepts (Brigandt 2010, following Brandom 1994). ${ }^{24}$ However, the class of materially valid inferences cannot be analyzed in terms of formal inference, since material validity contains that which is not captured by formal argument schemes (Brigandt 2010). The second reason is that material inference captures Wittgenstein's idea that meaning is (at least to some extent) use; we are able users of concepts and syntax without being able formulate the explicit rules for the use (if such exist at all). We can make materially valid, as well as formally valid, inferences even though we cannot always make the rule explicit that we follow in making such inferences.

Moreover, perhaps such rules do not even exist and some kind of "inferential particularism" is true. For more about "material inferences" see: Theron (1997), Brigandt (2010), Norton (2003).

\footnotetext{
${ }^{24}$ However, this has to be modified to: the class of inference that remain materially valid under almost every replacement, since otherwise it would contradict my claim that there are counter-examples to the universal validity of formal inferences, which would make my position internally incoherent.
}

(C) André Juthe. Informal Logic, Vol. 35, No. 3 (2015), pp. 378-445. 
Semantical paradoxes, on the other hand, are no reason against material inferences. The reason that semantical incoherences arise, is in my opinion due to the fact that our conceptual apparatus is fallible. We as fallible creatures can never in an exhaustively and perfectly manner mirror reality with our concepts (here the perspective of "critical realism" becomes relevant). Our concepts and the language thereof will lack perfect correspondence with reality. But that will neither contradict the general reliability of our material inferences nor of our concepts. However, no human logic or semantics will be without paradoxes.

Thus, all argument schemes are inferences entitled in virtue of truths embodied in concepts in a certain pattern. Substantial argument schemes employ material inferences: inferences by virtue of material content in factual and logical concepts. Formal argument schemes employ formal inferences: inferences by virtue of the "material content" in logical concepts alone (because not even logical concepts are completely devoid of material content).

\subsection{Formal, material and pragmatic validity}

Here I will define the notions of "pragmatic validity" and "material validity" in contrast to "formal validity" and explain what it means that an argumentation achieves "pragmatic validity." It would be inconsistent to define argument schemes with various inference configurations unrelated to formal validity and simultaneously claim that every argument must be formally valid. A "formal inference" is "formally valid" if and only if the justification of the inference is based solely on the meaning of the logical concepts of an argument scheme, whereas a "material inference" is "materially valid" if, and only if, the justification of the inference is based on the meaning of the various non-logical concepts of the argument scheme and not solely on the logical concepts. A material inference is justified by the factual content embodied in the concepts contained in the argument scheme and such schemes may have various degrees of strength.

An argument scheme is the inference configuration that enables the inference to take place and therefore also what ultimately defines what type of argument it is. I reject the notion of "neutral" or "negative" argument schemes. A legitimate inference configuration by the nature of the case, ceteris paribus, confers acceptability or plausibility to the standpoint, 
just like a formally valid argument-according to received opinion-that employs modus ponens with true premises will confer truth to the conclusion. Having an argument scheme which does not convey any acceptability to the standpoint presents itself as an absurd idea. An argumentation, that fails to transfer an increase of acceptability, to the standpoint, does not employ a legitimate scheme or make an inappropriate application of a scheme and is therewith an "invalid" argumentation.

An appropriately applied legitimate "argument scheme" means that the linking premise of the argumentation will make the "argument" if it is accepted-into a reason ceteris paribus, for the standpoint, whereas an incorrect or inappropriate applied argument scheme fails to accomplish that. This is the generic sense of 'validity.' Whether this reason is sufficient by itself for the standpoint, or whether it needs further arguments in order to accomplish that, is an open question that relates to the dialectical setting with respect to the concepts of acceptability, relevance and sufficiency. This is an important aspect because it explains why complex argumentation structures arise from a dialectical point of view. Additional arguments are the result of their dialectical function with respect to overcoming (anticipated) doubts or criticism to the "first" single argument.

This "generic validity" can be subdivided into the standard notion of "formal validity" and what I label "material validity." The latter can be further sub-subdivided according to which mode the inference operates; "conclusive" versus "inconclusive" inference. The terms 'conclusive' vs. 'inconclusive' and 'material' vs. 'formal' in this context are technical terms and only define the means and the mode by which the argumentation achieves transference of acceptability to the standpoint, and do not refer to any evaluation of the argument as such.

An argument scheme that has a conclusive mode makes the standpoint acceptable, ceteris paribus, to the same extent that the "argument" is acceptable, whereas the "argument" in an inconclusive scheme will increase the acceptability of the standpoint, but to a lesser degree than that of the "argument.",25

${ }^{25}$ More precisely: An argumentation has an inconclusively valid argument
scheme, if and only if, the "argument" in the scheme will increase the
acceptability of the standpoint, ceteris paribus, if the standpoint has or would
have, less acceptability than that of the "argument". An argumentation has a
conclusively valid argument scheme, if and only if, the "argument" in the
scheme will make the standpoint acceptability, ceteris paribus, to the same
extent as that of the argument, if the standpoint has less or would have less
acceptability than that of the argument. Formulated this way, the definitions
avoids counterexamples in which arguments used in favor of a standpoint

(C) André Juthe. Informal Logic, Vol. 35, No. 3 (2015), pp. 378-445. 
Argumentation with inconclusive argument schemes will conveys less "acceptability valence" than that of the "argument" to the standpoint. The argument with such a scheme will make it implausible that the standpoint is false if the "argument" is true.

The reason for the ceteris paribus clause for all types of inference configurations is that even argumentation with "conclusive" argument schemes can be defeated. There is a difference between refuting and defeating an argument, and while a sound deductive argument cannot be refuted it cancontrary to received opinion-be defeated (Tomic 2013). In other words, even a deductively valid and sound argumentation can still be defeated by additional information that yields a new conclusion that is preferred over the original (Tomic 2013).

This does not change the real contrast between conclusive and inconclusive "validity." There is a real difference between an argumentation that employs an inference which, unless defeated by additional information, confers a reason for a standpoint to the same extent that there is reason for the "argument," on the one hand, and an argumentation that employs an inference which, unless defeated by additional information, confers reason for the standpoint but to a lesser extent than there is reason for the "argument," on the other hand. Thus, any single argumentation with plausible premises regardless of whether it has "conclusive material validity" or "conclusive formal validity," does not make the argumentation into a conclusive reason for a standpoint, since its premises may be uncertain, or there may be counter-arguments against the standpoint or whatever.

In order to distinguish between argument schemes that can be defeated by underminers ${ }^{26}$ and those that cannot be defeated in that way, I introduce the notion of compactness, which means that the premises of the argumentation exclude the possibility that any additional premise may defeat the justification of the inference. The standard syllogisms of Aristotle may be examples of compact single argumentation.

All this means that the interpretation of an argument by identifying it according to what type it belongs is necessary prior evaluating its inference link. It does certainly matter how

which is already equally or more plausible than that of the "argument" in question.

26 There is a distinction between defeaters that defeat an argument by providing additional relevant information that undermines the inference link of the argument (labeled "underminers") and "overriders" which override the defense for a standpoint by attacking the standpoint with other arguments that trump (or equalize) the defense.

(C) André Juthe. Informal Logic, Vol. 35, No. 3 (2015), pp. 378-445. 
one formulates, interprets and classifies an argument with respect to evaluating its argumentative merits. I believe that argumentation that employs substantial argument schemes and are valid by material means, also can be of the "conclusive type." For instance, "arguments by conclusive analogy" is an example or such type of arguments.

The sub-subdivided materially valid argumentation into conclusive and inconclusive variants can in turn be sub-subsubdivided by which means the material validity is achieved: into the symptomatic, causal and analogical argument scheme.

In sum, that a single analogical argumentation in this sense is "conclusive" and "materially valid," means that the argumentation succeeds in being a reason, ceteris paribus, by means of an analogical argument scheme (which is a substantial scheme and therefore a material inference), for accepting the standpoint, to the same extent that there is reason to accept the "argument" of the argumentation. That a singular analogical argument is "inconclusive" and "materially valid" means that the argumentation succeeds in transferring acceptability from the "argument" to the standpoint and therewith increases its acceptability by means of an analogical argument scheme (which is a substantial scheme and therefore a material inference), but to a lesser extent than that of the acceptability of the "argument."

This distinction coheres very well with my previous distinction between argument by conclusive and argument by inconclusive analogy (Juthe 2005; 2009). An "argument by inconclusive analogy" is an argumentation that is "inconclusively valid" (in the sense defined in this paper) by material means, whereas an "argument by conclusive analogy" is an argumentation that is "conclusively valid" (in the sense defined in this paper) by material means.

An incorrect or inappropriate applied argument scheme fails to accomplish even the generic definition of "validity" or less than the argument type that its author was committed to. The same holds for deductive arguments that utilize formal validity as a criterion. Observe that 'validity' in any specified sense, is not defined in terms of relevance, rather the opposite holds: relevance is defined in terms of the validity of an argument scheme. A proposition argument $p$ is relevant for another proposition $q$ if, and only if, argument $p$ is a constituent of an argumentation that instantiates a "valid" argument scheme. And an argumentation with argument $p$ instantiates a material valid argument scheme if, and only if, $p$ succeeds being a 
reason, ceteris paribus for $q$, by substantial means. The reason that I choose that route is that I think that James Freeman is accurate in claiming that the type of relevance that connects propositions in an argumentation is primarily syntactical and not semantic in nature (Freeman 2011, pp. 142-148). ${ }^{27}$

The concepts of formal and material validity, however, should be supplemented with the concept of pragmatic validity. This is a dialectical notion that encompasses both material and formal validity and defines when an argumentation achieves transfer of acceptability from the perspective of the antagonist, and is consistent with the ten rules of the critical discussion. Thus, whereas the notion of material and formal validity are "objective," relating to the argumentation in itself, the notion of pragmatical validity is relative to the critical discussion. Hence, it follows that any argumentation that aims to be successful must be "pragmatically valid."

This also means that we can define when a fallacy is successful in deceiving a participant in a critical discussion, and when a fallacy is exposed as a fallacy. We can provide an extra dimension to our understanding of what a fallacy is. In the extended pragma-dialectical model, a fallacy occurs when the balance between the protagonist's goal of being reasonable is outweighed by the goal of being effective, and the protagonist's argumentation derails into violating one or more of the ten rules of the critical discussion (van Eemeren 2010). To this we can add that an argumentation that is "successful" in accomplishing effectiveness at the expense of reasonableness, is an argumentation which is pragmatically invalid, since regardless of whether it is formally or materially valid in itself, it could still violate one of the rules of the critical discussion (such as the unexpressed premise rule or the starting point rule) and fail to provide a reason for the standpoint in the critical discussion. ${ }^{28} \mathrm{~A}$ materially or formally invalid argument is, however, also automatically pragmatically invalid (it automatically violates one or more rules of the critical discussion).

\footnotetext{
${ }^{27}$ Note the resemblance of analyzing the "relevance" of the vertical relation in terms of a determining relation and this syntactical notion. A materially valid argument scheme "determines" a connection to the standpoint.

${ }^{28}$ For example, the argumentation could in a critical discussion beg the question and still be perfectly valid viewed by itself. (Rule 5 unexpressed premise rule: A party may not falsely present something as premise that has been left unexpressed by the other party or deny a premise that he or she has left implicit. Rule 6: No party may falsely present a premise as an accepted starting point, or deny a premise representing an accepted starting point. See van Eemeren et al. 2002, pp. 121-123, 128-130.)
}

(C) André Juthe. Informal Logic, Vol. 35, No. 3 (2015), pp. 378-445. 
The notion of "pragmatic validity" is fruitful in the system of pragma-dialectics and its idea of opposing parties in a critical discussion. The concept is particularly fruitful with analogical argumentation, since such "validity" will, as it should, depend on the background information of the participants of the critical discussion, which is obviously the case with argumentation by analogy. What the participants of the critical discussion consider relevant will be determined by the background information and what perspectives they have.

What you consider relevant will always depend on which theories and assumptions you have accepted. For instance, in an ethical discussion, judgments of relevance depend on what ethical viewpoints (say an utilitarian or deontological, etc.) the participants have, and while compared cases can be analogous from a utilitarian viewpoint, they could be completely disanalogous from a deontological point of view. Thus, a pragmatically valid argumentation is an argumentation in which an argument scheme succeeds in conveying the argument's accept-ability to the standpoint in a critical discussion, consistent with the rules of the critical discussion. Such an argument would probably also be successful from the viewpoint of the antagonist.

\subsection{Scheme-specific validity}

In the previous section I clarified the concepts of formal versus material validity as well as pragmatic validity. In this section I add another notion that relates to validity; scheme-specific validity, which means that validity is specific to each type of argument scheme. The concept of "scheme-specific validity" is a meta-description that qualifies every type of formal and material validity. Every type of scheme has its own type of "validity" in the sense that each scheme has its own standard as to when the inference connection is adequately established. If "validity" relates to the adequacy of the inference link and entitlement of the inference, the standard for this entitlement will vary according to the type and mode of the argumentation.

This is not as odd as it may appear; it is rather something that follows from the unified model of argument schemes outlined in the previous sections. It follows from the rather trivial insight that contrasting types of argumentation have contrasting standards of correctness conditions, as well as contrasting testing procedures. Given the generic definition of validity outlined in previous sections, you cannot judge an 
argument by standards of formal validity unless it is committed to a deductive interpretation. For example the argumentation:

Have you noticed that the lawn was wet? It was already wet so it must have been raining during the night.

could be interpreted as an attempt of single deductive argumentation:

1. It has been raining during the night;

1.1 The lawn is wet;

(1.1' If it rains, the lawn will be wet);

which would make it (formally) invalid as defined in this paper: The "argument," i.e., 1.1., does not transfer acceptability to the standpoint to the same extent that it itself is acceptable by means of the formal concepts of the argument scheme alone. However, if this argumentation is interpreted as an abductive argumentation ("inference to the best explanation"), the argument might be perfectly "valid" according to the scheme of abductive reasoning. Interpreted as an abductive argumentation, it fulfills the standard for inconclusive argument schemes (assuming a normal context): the "argument" 1.1 makes the standpoint more acceptable than without 1.1, but to a less degree than that of 1.1 itself. Hence the argumentation is "valid" if interpreted as an abductive argumentation.

This is does not mean that any formulation of a scheme will be valid "according to its type" so that it becomes meaningless to talk about "invalidity" at all or that any erroneous argumentation would be "valid" in "some sense." My point is that you cannot assess the "validity" of an argumentation unless you first have identified which type of argument scheme the arguer is committed to. A deductive single argumentation is an argumentation that is committed to employ a formal argument scheme that transfers the same acceptability as that of the "argument," ceteris paribus, to the standpoint, by means only of the logical concepts of the scheme.

In my theory, the criterion for validity is that the argumentation in question succeeds in achieving the specific standards of validity that belong to the type of scheme that the argumentation was committed to. Without this pragmatic aspect of the definition of argumentation, it would be impossible to discern a deductively invalid and a deductively valid argumentation, because you can always "play the enthymeme 
game" and supply a "gap-filling" premise and therewith making a formally invalid argument into a formally valid argument (Massey 1975).

While it is true that any argumentation can be reformulated into a deductive mode, there is a way that may help discern between "pseudo-deductive argumentation," which is an erroneously artificial reformulation of an argument scheme, on the one hand, and genuine deductive argumentation, on the other hand. The criterion is that the "argument" in a genuine non-deductive argumentation will transfer acceptability, ceteris paribus, to the standpoint even if it is not assumed to be formally valid, whereas a genuine deductive argument will not.

Thus, if the argumentation would be "generically valid" in a non-deductive formulation, then that is a reason against interpreting it, as a genuine deductive argumentation, ceteris paribus. This is not an absolute criterion, and should only be used when argumentative indicators by themselves are insufficient. Moreover, it cannot, for instance, be applied to abductive argumentation, because it is the reverse of deductive argumentation, and cannot therefore be discerned in that way. But the criterion can be a further indicator of whether an argumentation employs one of the three schemes of the pragmadialectical system or a deductive argumentation.

That is why I think many previous analyses of analogical argumentation have failed; they would have to be deductive in order to transfer acceptability to the standpoint, and therefore such argument schemes cannot be genuine cases of analogical argument schemes. The suggested model of analogical scheme advanced in this paper will transfer acceptability to the standpoint irrespectively of whether you (incorrectly) use a necessary background assumption as an unexpressed premise or not (see the logical minimum of my scheme in section [2.1]). However, transforming a genuine analogical argument into a formally valid argument contributes nothing to its argumentative merit; on the contrary, it obscures how the inference actually operates.

Moreover, as I argue in the next section, there are "invalid substantial schemes," such as can be seen in the example that attempts to reduce the analogical inference to a causal relation (the reader will understand what I refer to when he or she reads the next section).

The notion that I propose in this paper of a unified and generic material validity that is common to all arguments but that is specified into various types of validity for each 
substantial argument scheme has several advantages. First, the fact that all inferences have a common core of validity that is specified in various types of argument schemes makes it possible to have a unified classification of arguments. Arguments should be classified according to what essentially unites them and what essentially divides them into a nested hierarchy. This can be done with the kind of model that is presented here.

Secondly, this model does not violate our intuitions about how to reconstruct argumentation by squeezing arguments into formal validity where they all the same do not fit. Thirdly, the model also explains how deductive argument may be "conclusively valid," as well as how non-deductive arguments may be "conclusively valid," while simultaneously maintaining an essential difference between such arguments. It also explains the difference between various "inconclusive" argument schemes.

Fourthly, the model neatly solves the problem of enthymemes' inferences. The idea that all enthymemes should be changed into formally valid argument is problematic to say the least (Hitchcock 1998, see also Freeman 2011, pp. 173194). ${ }^{29}$ On this account, enthymemes are no particular problem because they have their own validity depending on what specific argument scheme each enthymeme employs in the reasoning. When context so indicates, one may conclude that enthymemes are elliptical versions of deductive argumentation, which yields an implicit premise that makes the argument formally valid; but in most cases they are elliptical versions of instances of some substantial argument scheme which yield an implicit premise that makes the argument material and pragmatically valid.

Finally, the model makes it much easier to pinpoint unexpressed premises when we are reconstructing argumentation (this is further discussed in section [4.2]). It is seldom easy to find the correct unexpressed premises, but the pragmadialectical idea that complex argumentation can always be broken down into single argumentation facilitates considerably

\footnotetext{
${ }^{29}$ Freeman contests and critiques Hitchcock's thesis that no enthymemes should be completed with additional premises into logically valid arguments - a critique I share. However Freeman also concedes that Hitchcock has a point in that most enthymemes should not be interpreted as elliptical argumentation; they are complete as they are. My point is that some enthymemes should be interpreted as elliptical versions of deductive argumentation whereas most should be interpreted as elliptical versions of argumentation that employ substantial argument schemes, and some are not even elliptical but should be taken at face value.
}

(C) André Juthe. Informal Logic, Vol. 35, No. 3 (2015), pp. 378-445. 
the search for unexpressed premises. Moreover, if you accept my suggestion of scheme-specific validity for each scheme, the task is made even easier; you have then the very narrow frame of a specific type of scheme and the number of plausible unexpressed premises that may make the argumentation valid (as defined in this paper) in accordance to its scheme becomes very small.

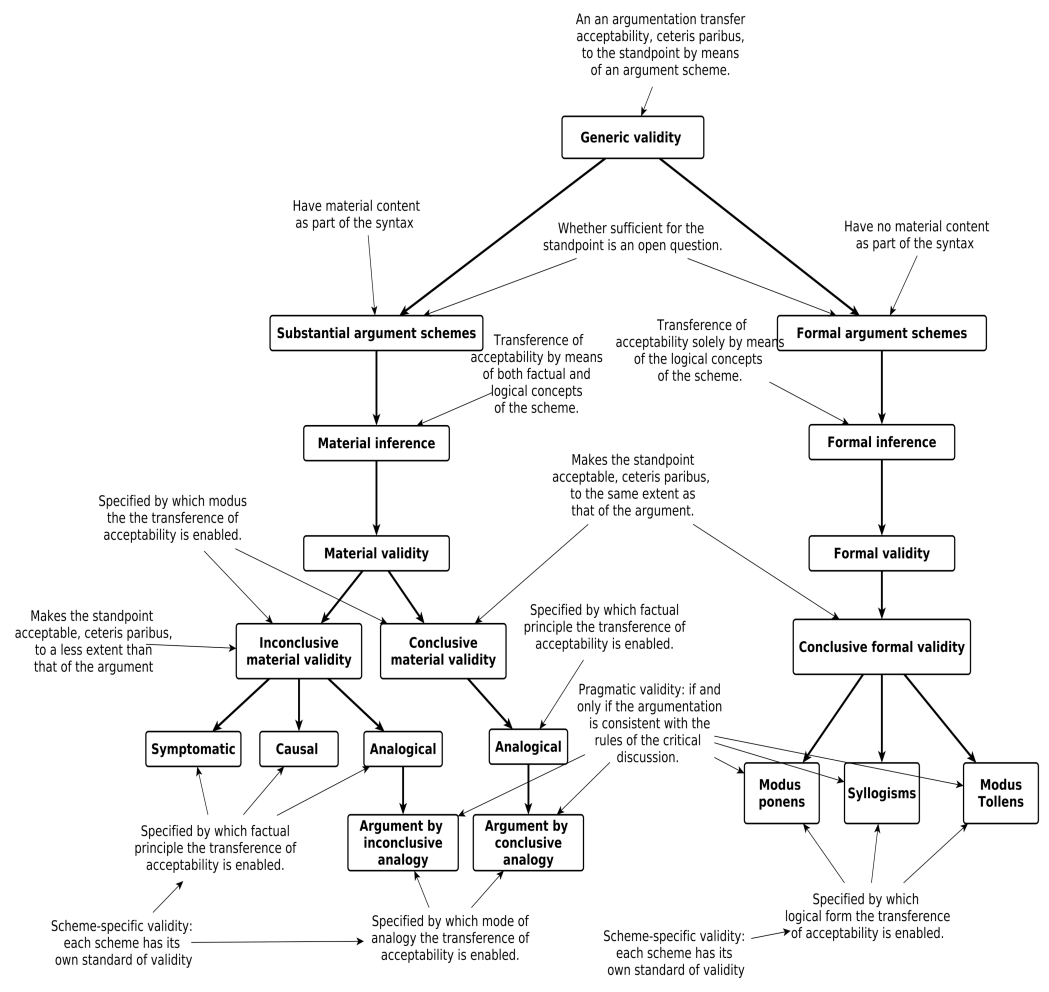

\section{Figure 2. The taxonomy of argument schemes and the} validity thereof

The idea of scheme-specific validity also has bearing on the "critical discussion." Consider a disagreement of opinion when the protagonist advances an argumentation with a symptomatic scheme. The antagonist, however misinterprets the argument as a deductive argumentation, and judges that the argumentation fails to confer truth to the standpoint, hence it is inconsistent with the validity rule. Thus, the argumentation of the protagonist is pragmatically invalid. However, the protagonist realizes the misinterpretation of the antagonist, and clarifies his argumentation, upon which the antagonist reconsiders the argumentation in accordance with the new standard of "validity" and now accepts that the argument does 
indeed provide a reason by means of a symptomatic relation. The argumentation is now pragmatically valid. An overview of the ideas expressed in section [3] can be seen in Figure 2, above.

\subsection{The analogical scheme is a genuine type of argument scheme}

In this section, I will argue that my analysis of the analogical argument scheme cannot be reducible to any other type of argument scheme in the pragma-dialectical system. One anticipated objection to my analysis in section [2] is that if there is this determining connection between the element and TargetSubject, then, in order to establish the claim Target-Subject has the Assigned-Predicate, all it takes is a premise describing this connection ("If an object has the element(s) $\in_{1} \ldots \in_{\mathrm{n}}$, then it has also the Assigned-Predicate ${ }_{\mathrm{AP}}$ ") and precisely the listing of elements for the target subject ${ }_{\mathrm{Ts}}$. The recourse to the analogous object "Analogue" would be superfluous; the argumentation is already valid (though perhaps not formally valid). However, then-depending on the degree of correlation-it is a probabilistic or deductive argumentation. It is no longer an argumentation by analogy based on a comparison.

We have already seen some of the problems with a deductivistic approach and I have elsewhere, with additional reasons, shown that such deductive reformulation is a mistake (Juthe 2005, 2009). I will therefore only focus on the objection that analogical argument schemes can be reduced to some causal or symptomatic argument scheme. Another reason is that the argument schemes in the pragma-dialectical perspective are defined by different principles than the standard categories of abductive and inductive inferences and cut across such categories (some inductive reasoning have causal schemes, others have symptomatic, and the same cross-categorical taxonomy holds for abductive argumentation). I will therefore only discuss the typology in the pragma-dialectical system.

The question is, can argumentation that is assessed as a genuine analogical argumentation by argumentative indicators, always be reduced to a causal or symptomatic argumentation?

Such reduction cannot be done if the analogical argument scheme has some essential feature that no other scheme has, and there is such an essential difference. The essential difference between an analogical argument scheme and any other inference configuration is that analogical inference is same-levelreasoning, whereas all other schemes are different-level- 


\section{André Juthe}

reasoning. Analogical inference is inference via resemblance between particulars, not inference by a general to a particular or vice versa. All other schemes (including inductive-deductive patterns) move across contrasting levels. All other inference schemes proceed via a general to a particular or via a particular to a general. ${ }^{30}$ However, there is no need to know a category over and above the particular in an analogical predicate transfer.

Furthermore, we infer the vertical determining relation unto the Target-Subject by virtue of the horizontal one-to-one relation. We project the determining relation because we perceive and recognize that there are counterpart elements in the target-domain. The vertical determining relation is never known independently of the "act of perceiving resemblance."

Thus, we cannot use the vertical determining relation without knowing about the comparison of resemblance between particulars. This is very clear when we look at concrete examples of argumentation that employ different-domains analogical schemes. For instance, the single argumentation by analogy:

Just as we cannot adequately conceive of red otherwise than in terms of red experiences, positive value also cannot be so conceived apart from mentioning responses of moral approbation on our part. (Tropman 2010: p. 35) ${ }^{31}$

can be reconstructed:

1. Positive value $\mathrm{TS}_{\mathrm{TS}}$ cannot be adequately conceived apart from responses of moral approbation AP*. .

1.1 The experience of red $_{\in}$ of adequately conceiving of $\operatorname{red}_{\mathrm{A}}$ is comparable to our response of moral approbation $_{\in} *$ in conceiving positive value $\mathrm{TS}_{\mathrm{T}}$ [comparison]

(1.1' The experience of red $_{\in}$ is essential for us to adequately conceiving red $_{\mathrm{AP}}$.) [determining relation]

\footnotetext{
${ }^{30}$ While I will not discuss abductive, conductive or inductive arguments, I am convinced that this holds also for argument that is classified as such arguments by the standard approach. Deductive, inductive and abductive are all different-level-reasoning. For instance, an enumerative induction like swan 1, swan 2, swan 3 are all white, hence it reasons from the particulars 1, 2 and 3 to the general category of swans in general.

31 The argument was Elizabeth Tropman's attempt to describe John McDowell's argument for the sensibility theory in meta-ethics.
}

(C) André Juthe. Informal Logic, Vol. 35, No. 3 (2015), pp. 378-445. 
This argumentation cannot be reduced to causal or symptomatic scheme for two reasons. First, it does not move across contrasting levels of reasoning: It neither moves from knowledge of general symptoms to a particular, nor via a general knowledge of causes to a particular effect; rather, it moves from knowledge about one particular-how we must conceive of red (the Analogue) - to another particular-how we must conceive of value (the Target-Subject).

Secondly, knowing 1.1' is obviously insufficient to entitle any support for 1.; and 1.1' needs the resemblance expressed in 1.1 in order to transfer any acceptability to 1 . Thus, the assigning of the Assigned-Predicate to the Target-Subject cannot be done without the horizontal relation of comparison with the Analogue. The vertical determining relation expressed in 1.1' cannot be known to hold in the Target-Subject without the resemblance expressed in the comparison. Thus, the act of perceiving the one-to-one correspondence is an essential part of the inference. The inference that the same determination (i.e., "essential") holds between positive value and moral approbation is entitled by the comparison to how our experience of red is essential for adequately conceiving red. The justification of a corresponding vertical determining relation in the TargetSubject is by virtue of the resemblance with the Analogue.

The reasons against the idea that analogical argument schemes understood according to the theory outlined in section [2], can be reduced to some other scheme, are clear when you try to perform it on different-domains analogies. It becomes, however, even stronger when you look at complex argumentation by analogy since the vertical and the horizontal relation as a whole is then built of a compound of single arguments that operate in unified fashion. This means that the vertical and horizontal relations are composed of several subrelations. Take a look at a different-domain argument by conclusive analogy by C.S Lewis:

You can get a large audience together for a strip-tease act - that is, to watch a girl undress on the stage. Now suppose you came to a country where you could fill a theatre simply by bringing a covered plate onto the stage and then slowly lifting the cover so as to let everyone see, just before the lights went out, that it contained a mutton chop or a bit of bacon, would you not think that in that country something had gone wrong with the appetite for food? (Lewis 1952, p. 75) 


\section{André Juthe}

Could you really reduce this to some non-analogical argumentation? This argumentation can, without problem, be reconstructed as a complex made of two single analogical arguments that lend a subordinative defense of the linking premise (1.1'):

1. Something has gone wrong with the appetite for sex in today's society.

1.1 Something has gone wrong with the appetite for food in the hypothetical country.

1.1' The appetite for sex in todays society is comparable to the appetite for food in the hypothetical country.

1.1'.1a Today's crowded striptease shows are comparable to filled theatres showing a plate with food onto the stage in the hypothetical country.

(1.1'.1a' A filled theatre with people that want to see a plate with food onto the stage is relevant for the judgment that something gone wrong with the appetite for food in the hypothetical country.)

1.1 '.1b The strip-tease act (to watch a girl slowly undress on the stage) is comparable to having a covered plate onto the stage and then slowly lifting the cover so as to let everyone see, just before the lights went out, that it contained a mutton chop or a bit of bacon.

(1.1'.1b' Having covered plate onto the stage and then slowly lifting the cover so as to let everyone see that it contained a mutton chop or a bit of bacon is relevant for the judgment that something gone wrong with the appetite for food in the hypothetical country.)

Each single argument by analogy adds one element of resem-blance in a cumulative coordinatively compound argumentation for the major linking premise 1.1'. Moreover, each single argument has a proposition ("argument" 1.1'.1a and 1.1 '.1b) that expresses the horizontal relation between the elements, and a proposition which express the vertical determining relation (linking premise 1.1'.1 a and 1.1'.1 b'). Thus, the argumentation can readily be reconstructed according to the theory of analogical argument schemes outlined in section [2]. But how do you reconstruct it if you must employ some other 
argument scheme? And even if you could reconstruct it along some other scheme, could you do it in a way that did justice to the pre-reconstructed argumentation?

The logic of correctly reconstructing complex analogical argumentation will be discussed in the next section. However, what can be pointed out here is the futility of thinking that the vertical determining relation could operate as an inference license without the horizontal one-to-one relation of comparison. The futility of such an endeavor becomes painfully clear when the analogical argument employs a complex different domain analogy, as in this case. How could the fact that a theatre filled with people that want to see a plate with food on the stage, and having a plate covered with a mutton chop, determine that something has gone wrong with the appetite for food-have any inferential connection with the appetite for sex in today's society-without the one-to-one correspondence relation of resemblance with today's strip-tease shows?

The only way to make the determining relation part of an inference configuration that is not analogical would be to reformulate it into an exceedingly dubious and far-fetched and highly abstract universal premise and turn the argument into a deductive argumentation. A move that would both fail to do justice to the pre-reconstructed argument and be much worse off as an argument just as the deductive formulation of the inductive argument discussed in the beginning of this section was worse off as an argument.

Lewis's argument is essentially a comparison between two very distant things, and attempts to find a specified universal premise that includes exactly those features that are relevant, and that excludes exactly those features that are irrelevant and that excludes exactly all those features that may be negative relevant (all of which is necessary in order to be a deductive argument that avoids obvious counterexamples) are just doomed to failure. Thus, this example not only shows that you cannot reduce an analogy to a symptomatic or causal scheme but constitutes a real bite against deductivism as well. (An even more formidable example of irreducible argumentation by analogy is provided in section [4].)

The same irreducibility holds with argumentation by analogy that employs same-domain analogies, though it is not equally clear. I will therefore go deeper into an example of same-domain analogy. For example the argumentation we looked at in section [2.1]: 
The Porsche and the Chevrolet are both in the $\$ 40,000$ price range, and the Porsche is of excellent quality. Therefore, the Chevrolet is probably also of excellent quality. ${ }^{32}$

is not reducible for the same reasons. The vertical determining relation never says that element $\$ 40,000$ price range $\in$ in general or that for every object that has such price range it will also have excellent quality ${ }_{\mathrm{AP}}$. For instance, the argumentation does not license the inference on basis of "If a car cost $\$ 40,000$, then it has excellent quality." Rather, it compares a Porsche with a Chevrolet and claims that since they are similar with respect to price, which is relevant for quality, they will also be similar with respect to quality. The point is that it (implicitly) claims that the Porsche's price range is relevant for the Porsche's excellent quality (not any car) and that the Chevrolet is similar to the Porsche with respect to price range as a reason to believe that also the Chevrolet is of excellent quality. You have no warrant to disconnect knowledge of the determining relation from knowledge about the Porsche and therefore you need knowledge about the resemblance in order to extend the same determining relation in the case of the Chevrolet.

Moreover, as we shall see in what follows, you get into various difficulties when you try to interpret the scheme as nonanalogous. The best reformulation with a symptomatic argument scheme yields: ${ }^{33}$

1. The Chevrolet will probably be of excellent quality

1.1a The Chevrolet is in the same price range as the Porsche

1.1b The Porsche is of excellent quality

(1.1a-1.1.b' Cars in the same price range generally have the same quality)

1.1a.1 The Porsche and the Chevrolet are both in the $\$ 40,000$ price range

${ }^{32}$ In a previous work (Juthe 2005) it is claimed that this argument was a distinct type of "argument by similarity" and could not be considered an argument by analogy. I have changed this conviction since I think that implicitly even the most simple argument by comparison which compares just one property with another property in the Target-Subject in a broad sense has a vertical relation.

${ }^{33}$ This reconstruction was suggested by Francisca Snoeck Henkemans. 
While this interpretation is certainly not completely unreasonable, and would render the argument materially valid (and probably pragmatically valid as well) as defined in this paper, it does not do full justice to the pre-reconstructed text. ${ }^{34}$ Furthermore, the reconstruction does not really make sense from a pragma-dialectical perspective. Reconstructed this way means that it is a coordinatively compound argumentation where $1.1 \mathrm{a}$ and its linking premise is the "first" argumentation and $1.1 \mathrm{~b}$ is an addition. But what would be the dialectical function of $1.1 \mathrm{~b}$ if interpreted as a coordinative move? How could 1.1b help 1.1 a overcome doubts or refute an anticipated counter-argument against the sufficiency of 1.1a? It make no sense in that respect. Rather, 1.1b looks like the linking premise for 1.1a. Moreover, why would it be important to state they are "both in the $\$ 40,000$ price range" and that "the Chevrolet is probably also of excellent quality," in the pre-reconstructed text, unless they were compared? Why bother to mention the Porsche at all? Why not just state it as follows?:

1. The Chevrolet will probably be of excellent quality.

1.1 It is in the $\$ 40,000$ price range.

$1.1^{\prime}$ Cars that are in the $\$ 40,000$ price range generally have excellent quality.

While you could argue that the fact about the Porsche is used as a subordinate defense:

1.1'1 The Porsche is in the $\$ 40,000$ price and has excellent quality.

as in the first interpretation, it would make the interpretation violate the principle of charity yielding a very weak defense (using only one example as evidence of a general proposition arguably commits the fallacy of hasty generalization).

Finally, the proposition that would express the vertical determining relation in an analogical interpretation (1.1'1 in this case) does not perform any inferential work in this nonanalogical reconstruction. In this reconstruction, it performs a dialectical function of lending subordinative support to the claim that the price range correlates with excellent quality. Thus, the vertical determining relation does not perform the inferential

\footnotetext{
${ }^{34}$ In order to be formally valid the linking premise has to read: "All cars in the same price range have the same quality," or "If cars have the same price range, then they also have the same quality."
}

(C) André Juthe. Informal Logic, Vol. 35, No. 3 (2015), pp. 378-445. 


\section{André Juthe}

work it ex hypothesis would do if analogical inferences are reducible to other kinds of inference configurations. Just claiming that a pre-reconstructed argumentation may be interpreted in another way, is not equivalent to claiming that an argument scheme can be reduced to another type. Other reconstructions that would employ a linking premise like "all cars in the $\$ 40,000$ price range typically are of excellent quality," will have worse problems, making the information about the Porsche completely irrelevant.

Notice furthermore that the linking premise 1.1' in a nonanalogical interpretation expresses a category over and above the particular Chevrolet and that you need to know something about a general category, i.e., that "'cars in the same price range generally have the same quality," which you in the actual argument have no indication of knowing. In contrast, what you do have an indication of knowing in the actual pre-reconstructed argument, is knowing something about a particular, i.e., that "the Porsche that is in the $\$ 40,000$ range has excellent quality" and knowing something about another particular: "the Chevrolet is also in the $\$ 40,000$ range." Furthermore, the claim about the Porsche becomes irrelevant to the inference configuration of the argument regardless of how you formulate the argument in a non-analogical reconstruction. The causal argument scheme suffers an even worse fate. The least problematic reconstruction gives:

1. The Chevrolet is probably of excellent quality.

1.1 Both the Porsche and the Chevrolet are in the $\$ 40,000$ price range.

1.1' A $\$ 40,000$ price range causes the Chevrolet to have excellent quality.

which at least yields a materially valid causal argument scheme and also makes the ex hypothesis "vertically determining relation" do the inferential work. However, it makes half of the information in 1.1. redundant and irrelevant. Besides, 1.1' strikes one as counter-intuitive, since it is rather the other way around: it is rather that excellent quality causes price range than vice versa. However, a formulation that "excellent quality causes price range" would make the argument generically invalid as defined in this paper; the scheme would fail to ceteris paribus transfer acceptability - to any extent - to the standpoint.

Finally, as stated in section [2], an analogical inference may be based on intuitive judgments of similarity without 
knowing either exactly what the vertical determining relation is, or exactly what the horizontal corresponding relation is. They are part of the ontological correctness conditions for arguments by analogy, not necessarily the conditions for being able make the inference. An argument is committed to the existence of the ontological correctness conditions, but it can be a good argument without knowing exactly what they are. Thus, you do not need to always know exactly about the detailed structure of an analogy in order to employ it in an argument, just as you do not need to know every background assumption or unexpressed premise for a deductive argument in order to assess its validity. Thus, while an argument by analogy cannot operate without the crucial relations and constituents outlined in section [2] and be materially valid (as well as pragmatically valid), you do not need to be able to explicitly spell them out.

There is an important difference between gap-filling unexpressed premises and tacit background assumptions and there are various types of such assumptions (Govier 1972; Ennis 1982; Gough and Tindale 1985; Holtzman 1997; Plumer 1999; Gerritsen 2001). There are a lot of assumptions that are necessary in order for arguments to work, but which do not fill gaps in the inference - the standard role for missing premises. A reconstruction of an argument's unexpressed premises must be acceptable to both parties in a critical discussion, and make the argumentation pragmatically valid (as defined in this paper). The question is then, if this is correct, how should one correctly reconstruct complex argumentation by analogy? That is a question that will be answered in the next section.

\section{The reconstruction of complex analogical argumentation}

I have hitherto focused primary on the microstructure of analogical arguments and have only to a minor extent talked about the macrostructure of argumentation by analogy. I have discussed the inference configuration of analogical argument schemes and how it pertains to the meaning of 'validity' and 'inference' in the larger context of argumentation theory as a whole. In this section, the focus will instead be upon the macrostructure of analogical argumentation and we will take a look on how to reconstruct argumentation by analogy that has a complex argument structure. I order to do this fully, I need to accomplish three things. I need to clarify the notion of dialectical vs. logical argumentation structure. This is done in section [4.1]. I need to explain how to find unexpressed premises in this 
system, which is done in section [4.2]. I will also need to apply my theoretical model of analogical argument schemes on a real life concrete example and show the result thereof. This is done in section [4.3].

\subsection{Dialectical versus logical structures of argumentation}

Before I discuss complex analogical argument structure, I need to clarify and distinguish between the notions of dialectical versus logical argumentation structure. The pragma-dialectical tradition distinguishes single and complex argumentation structures, and an important tenet of pragma-dialectics is that all types of complex argumentations are constructed out of a combination of single arguments. Thus, complex argumentation can always be broken down into a number of single arguments (van Eemeren et al. 2002 pp. 63-75; Snoeck Henkemans 1997, 2000).

The complexity of complex argumentation structure according to the pragma-dialectical paradigm is the result of the dialectical function of responding to critical reactions. While the simplest type of argumentation consists of only one single argument, a complex argumentation can be complex in three basic ways: Multiple argumentation consists of more than one sufficient separate defense of the same standpoint. ${ }^{35}$ Subordinatively compound argumentation consists of a serial chain of arguments in which each argument supports the preceding argument up to the standpoint. ${ }^{36}$ Coordinatively compound argumentation consists of several single arguments that coordinatively cooperate in order to constitute a sufficient defense of the standpoint. ${ }^{37}$ These three basic variants may be combined endlessly, which makes the level of complexity theoretically unlimited. Each structure also has different indicators, which provide clues as to which type of argument structure the arguments have constructed (Snoeck Henkemans 1997, pp. 129-167; 2003; 2010; Eemeren, Houtlosser \& Snoeck Henkemans 2007).

The school of informal logic has also three main contrasting argument structures; serial, linked and convergent, where the serial is equated with subordinative, linked with

35 The dialectical function of multiple argumentation is to provide an alternative sufficient defense if the "first" argument was rejected.

${ }^{36}$ The dialectical function of subordinative compound argumentation is to provide a defense of the acceptability of the "first" argument.

37 The dialectical function of coordinative compound argumentation is to provide a defense of the sufficiency of the "first" argument.

(C) André Juthe. Informal Logic, Vol. 35, No. 3 (2015), pp. 378-445. 
coordinative and convergent is equated with multiple argumentation. However, the reader should be aware that these two clusters of distinctions are made from different disciplinary perspectives, and therefore cannot be equated. To confuse the dialectical structure with the logical structure of an argumentation is to confuse different things. Some authors have erroneously thought that there is just terminological difference, which is not the case (see for example Walton 1996, pp. 114115). The pragma-dialectical paradigm analyzes argument structures from a dialectical perspective, whereas informal logic analyses from a logical perspective. From a pragma-dialectical perspective, argument structures result from various dialogical exchanges aimed at resolving a difference of opinion, whereas from the perspective of informal logic it is the logical relations between the "arguments" ("premises" in the terminology of informal logic) that determine which structure they have. Subordinatively compound argumentation in Pragma-dialectics results from the protagonists effort to overcome doubts or criticism of acceptability, whereas serial argumentation in informal logic holds because the premises lend support to the previous in a serial chain.

Coordinatively compound argumentation in Pragmadialectics exists because the protagonist responds to doubts or attacks on sufficiency of an argument, whereas argumentation in Informal logic is linked because the premises in an argument share a mediating link (Freeman 2011, pp 159-191). ${ }^{38}$ In pragma-dialectics an argumentation is multiple because the protagonist wants to have more than one separate alternative defense of the standpoint whereas in Informal logic, convergent argumentation occurs because each premise of the argument is an independent reason for the conclusion, i.e., without any intermediate concept (Freeman 2011, pp 159-191).

Convergent argument structure means that each "argument" (premise) does not need any other "argument" (premise) to be a reason for the standpoint.

However, even if the perspectives are from dissimilar disciplines, it does not follow that they are logically independent of each other. Just like certain biological facts will entail certain chemical facts and vice versa, the dialectical dimension will have implications for the logical dimension and vice versa.

The distinction between convergent and linked argument-

\footnotetext{
${ }^{38}$ There is a controversy as to how exactly distinguish between linked and convergent argumentation structure and what criterion should be used. I have decided to follow the criterion of James Freeman (Freeman 2011).
}

(C) André Juthe. Informal Logic, Vol. 35, No. 3 (2015), pp. 378-445. 
ation, has been given various criteria but they have all been problematic (Walton 1996). The distinction by Freeman is based on the difference between relevance linkage and modal "linkage" (Freeman 2011, pp. 94-103). Relevance linkage entails a linked argumentation, whereas a completely separate argument or with only modal "linkage" entails a logically convergent structure. 'Modally linked' means that they each on their own add to the support for the standpoint (Freeman 2011, pp. 94-103). In turn, the criterion for discerning between relevance linkage and modal linkage is, according to Freeman, whether they have a mediating concept in the premises that does not occur in the conclusion (Freeman 2011, definition on p. 139). ${ }^{39}$ A mediating concept implies a relevance linkage. This make sense, the concept of linkage should from a logical perspective signify a logical, that is, syntactical connection in the logical structure.

Accepting Freeman's criterion means that an argumentation that has "modal linkage" has convergent argument structure and an argument that has "relevance linkage" has linked argument structure. That means that dialectically complex argument structures like multiple or cumulative coordinatively compound argumentation will be logically convergent. A Cumulative Coordinatively compound argumentation structure cannot have a logical structure of relevance linkage, since "doubting sufficiency" presupposes that the antagonist consider that the doubted argument was at least relevant for the standpoint in question. From a logical point of view, they share no mediate concept, therefore they are only modally "linked" and therewith independently relevant for the standpoint, hence they are convergent (if assuming Freeman's criterion). Single argumentation in the pragma-dialectical system will, from a logical point of view, always have linked argument structure since all propositions are necessary in order to be a reason at all.

Even though an argumentation structure with either contrasting types of argument schemes or contrasting instances of the same type of argument scheme is a necessary condition for being a multiple argumentation structure, it is not a sufficient condition. Each argument must also be sufficient on dialectical grounds before you can claim that they have a multiple argument structure. Whether the protagonist thinks that each by itself is a sufficient defense for the standpoint in the critical discussion is an assessment of the context and various argumentative clues (Snoeck Henkemans 1997).

\footnotetext{
${ }^{39}$ For a critical evaluation of Freeman's criterion, see Hitchcock (2015).
} 
From a pragma-dialectical perspective, where the assessment of argumentation structures is dialectical, the question of acceptability emerges in connection with reasons which may consists of many premises, whereas in a logical assessment it emerges with premises. If you have to evaluate an argumentation where you have a mix of convergent and linked structure, it is particularly important to discern which premises need to be taken together to compose one reason and which premises constitute a reason by themselves (Freeman 2011, makes this point p. 112). Thus, even if you reconstruct an argument structure by dialectical criteria, you still need a logical approach as well.

However, to think that any of these disciplinary perspectives is better or more truthful than the other, would be like thinking that biology is a better or more truthful perspective than chemistry. Rather, they are both necessary in order to fully depict and understand the whole realm of argumentation. While argumentation structures of these contrasting disciplinary perspectives often coincide, sometimes they do not. For instance an argumentation like this:

(1) Bart is $179 \mathrm{~cm}$ tall.

(2) André is $181 \mathrm{~cm}$ tall.

(3) Frans is $180 \mathrm{~cm}$ tall.

(4) Francisca is $178.5 \mathrm{~cm}$ tall.

(5) Jackie is $179.5 \mathrm{~cm}$ tall.

(6) All five researchers are approximately equally tall.

would, in the pragma-dialectical system, be an instance of single argumentation whereas it would count as "complex" and linked argumentation in the system of informal logic. Moreover, coordinatively compound argumentation of the complementary version cannot be handled at all from a purely logical perspective. For instance, the structure of this argumentation:

I think we should buy this book, then we have at least something to give him, and he can always exchange it. ${ }^{40}$

makes perfect sense from a dialectical perspective and should be reconstructed as a complementary coordinatively compound argumentation: ${ }^{41}$

\footnotetext{
${ }^{40}$ The example is taken from Snoeck Henkemans (1997, p. 169).

${ }^{41}$ The reconstruction is from Snoeck Henkemans (1997, p. 170). 
1. We should buy this book for him.

1.1a Then we have at least something to give him.

$1.1 \mathrm{~b}$ He can always exchange it.

The reason $1.1 \mathrm{~b}$ is part of the argumentation is for dialectical reasons as means of refuting the anticipated objection- "we don't know if he already has the book" - against the sufficiency of 1.1a.

The question is, what logical structure does this argumentation have? When we add the unexpressed linking premises to the argumentation, we have:

1. We should buy this book for him.

1.1a Then we have at least something to give him.

$1.1 \mathrm{a}^{\prime}$ We should have something to give him.

$1.1 \mathrm{~b}$ He can always exchange it.

$1.1 b^{\prime}$ If he can exchange it, it will not do anything if he already has it.

We see that each argument in the argumentation employs two contrasting deductive schemes (modus ponens and modus tollens) ${ }^{42}$ Note that $1.1 \mathrm{~b}$ fails to be relevant for 1 , since $1.1 \mathrm{~b}$ in conjunction with $1.1 b^{\prime}$ does not constitute a reason, ceteris paribus, for 1 to the same extent as $1.1 \mathrm{~b}$ is true. Remember that relevance was defined in terms of argument scheme and that the specified sense of validity of deductive arguments was conclusive formal validity: the argument scheme ensures a reason for the standpoint to the same extent as the "argument" is true, by solely formal concepts of the scheme. However, the arguments (premises) are not linked; they share no mediating link that creates a relevance linkage.

Furthermore, the argumentation cannot be defined as convergent because the arguments do not converge on the same standpoint: $1.1 \mathrm{~b}$ refutes the objection that he might already have the book by supporting another proposition: "It will not do anything if he already has the book" which is a completely different proposition than that of the standpoint. But this means that this is an example of an argumentation-which obviously is not serial-but which has a structure that does not make sense from the perspective of logic; it is neither convergent nor

\footnotetext{
${ }^{42}$ It does not make sense to interpret either $1.1 \mathrm{a}$ or $1.1 \mathrm{~b}$ as employing any of the substantial schemes. 1.1a is interpreted as: "If we don't buy this book, we have nothing to give him."
}

(C) André Juthe. Informal Logic, Vol. 35, No. 3 (2015), pp. 378-445. 
linked. ${ }^{43}$ Examples like this also give us a reason to accept the pragma-dialectical distinction between schemes and structure.

This phenomenon will strike at any attempt to handle complementary coordinatively compound argumentation from a logical perspective. Thus, the dialectical perspective is necessary and you cannot explain certain argumentation without it.

Subordinatively compound and serial argumentation are, however, coextensive. The logical structure of argumentation that has a dialectically subordinative argument will always be serial, even though they are reconstructed from dissimilar perspectives. It should be emphasized that since the main goal of this paper is to show how a theory of analogical argument scheme fits with the pragma-dialectical system, the complex analogical argument structure in this paper concerns the dialectical argument structure, not the logical. However, regardless of whether you want to reconstruct a complex argumentation from a logical or dialectical perspective, you need to fill in unexpressed premises to make the argumentation complete and identify what scheme the argumentation utilizes, and that brings us to the next section.

\subsection{The search for the unexpressed premise}

In order to properly reconstruct an argumentation, the concept of unexpressed premises becomes pressingly acute. The reconstruction of complex argumentation by analogy is no different in that respect. Since there are many tacit elements that

\footnotetext{
${ }^{43}$ One could object that $1.1 \mathrm{~b}$ is weakly relevant for 1 , in that $1.1 \mathrm{~b}$ would constitute (a very odd indeed but nevertheless) a reason for 1 (and therewith $1.1 \mathrm{~b}$ is converging on 1 after all). However there are three reasons against such view. First it would not distinguish between broad and narrow relevance. Topical relevance of argumentation should be narrowly relevant (clearly $1.1 \mathrm{~b}$ does not seem relevant as a direct reason for 1 without 1.1a.) even though it is not completely unassociated to the topic in a broad sense (i.e 1.1b is not irrelevant in the same sense as, say, giving the color of the sun as a reason for 1.). Secondly, if we accept such a logical structure then we are missing the dimension of the dialectical function of the coordinative addition that $1.1 \mathrm{~b}$ really is, and should not the logical structure express what $1.1 \mathrm{~b}$ primary is supporting (i.e., "It will no do anything if he already have the book")? The "support" (if it can be said to be that) of $1.1 \mathrm{~b}$ for 1 is more of an unintentional byproduct and not the reason for why $1.1 \mathrm{~b}$ is part of the argument structure. Finally, if $1.1 \mathrm{~b}$ is relevant for 1 , it has to be part of an argument scheme that is generically valid (in the sense defined in this paper) but the only way it can be made valid in a reasonable way is to make it into a deductive argument, and the only way to make $1.1 \mathrm{~b}$ formally valid for 1 . is to have the linking premise to state: "If he can exchange it, we should buy it for him" an obviously implausible proposition in this context.
}

(C) André Juthe. Informal Logic, Vol. 35, No. 3 (2015), pp. 378-445. 
play a role in argumentation, it is not easy to find the correct unexpressed premise (Gerritsen 2001 makes this point pp. 9495). As noted earlier, unexpressed premises are just a subset of all implicit assumptions, and there are many propositions that function as background assumptions without having the function as premises of an argument (Govier 1972; Ennis 1982; Gough and Tindale 1985; Holtzman 1997; Plumer 1999; Gerritsen 2001). The function of unexpressed premises is to fill a gap in the inference whereas background assumptions may have all kinds of functions necessary for the argument.

Some philosophers have made a distinction between used versus needed premises; the former being the premise that was actually used in an argumentation whereas the latter being what logically must be there for the argument to be formally valid (Ennis 1982). Obviously, it is much more difficult to discern what premise an interlocutor actually used and what he perhaps was not even aware of himself, than assessing what an argumentation needs to assume in order to be valid.

However, here again the concept of "validity" becomes critical and you will not find the correct unexpressed linking premise if you automatically search for a premise that would make the argumentation formally valid. Instead, I suggest scheme-specific validity for each type of argumentation, which was presented in the previous section. Having as narrow a frame as that of a well defined argument scheme of a single argumentation makes it much easier to determine the unexpressed premise. You will know what you are looking for in a very limited set of candidates. Van Eemeren and Grootendorst (1984), stated that the proposition identified as an unexpressed premise must fulfill certain conditions (p. 141):

1. It must make the argument valid. (Relation)

2. It must be informative. (Quantity)

3. It must be an element of the speaker's committedness. (Quality)

I think this is approximately correct, but it can be further refined in particular with respect to 1. First of all, the first conditions should not be interpreted as requiring formal validity. I suggest the following procedure to find the linking premise:

(1) Determine which argument type the arguer is committed to (by examining argumentative indicators). 
(2) Determine which proposition makes the argument 'valid' according to the type of scheme it employs and in the sense of valid as defined by the scheme (as 'valid' is defined in this paper).

(3) Determine which proposition in the set of alternative propositions that satisfies (1) \& (2) is the most informative and least trivial.

(4) Determine which proposition in the set of alternative propositions that satisfies (1) \& (2) \& (3) is the least abstract, i.e., makes the least ontological commitments to entities appropriate to the context. ${ }^{44}$

In order to determine (1), you need to know which indicators are used for each type of argument scheme and from that you can identify what is necessary for the argument scheme. Neither the logical minimum nor the pragmatical optimum, ${ }^{45}$ but rather what the protagonist is schematically committed to, is what should be sought for.

The unexpressed linking premise is defined as that proposition that is needed in order for a scheme to be valid in accordance to its type. (as 'valid' is defined in this paper). Its function is to entitle the inference and there are a limited set of propositions that could entitle the inference in a particular type of argument scheme instantiated by a particular argument in a particular context.

This linking premise can be distinguished from other assumptions necessary for the argument, as background assumptions, but which do not have the function of enabling the inference. The "linking premise" is unique in that it constitutes the link between the "argument" and the standpoint and entitles the inference. The linking premise defines what type of argument scheme the argumentation has instantiated because a scheme is defined by which means it transfer the acceptability of the "argument" to the standpoint.

\footnotetext{
44 "Appropriate to the context" means that sometimes you are entitled to interpret a wider ontological commitment than a narrow interpretation. For instance, if you discuss the price of cars like Volvo and Saab and employ symptomatic argument schemes, then the context may entitle you to attribute a generalization over cars in general to the argumentation, and not only over these particulars brands.

45 The "pragmatic optimum," in contrast to the "logical minimum," is a premise that makes the argument formally valid and also prevents a violation of a rule of communication. The pragmatic optimum is as informative as possible without imputing unjustified commitments to the speaker, and formulates the premise in a informal way that suits the discourse as a whole.
}

(C) André Juthe. Informal Logic, Vol. 35, No. 3 (2015), pp. 378-445. 
Therefore the linking premise is in some sense the essence of an argument scheme. Thus, I think that the distinction between used and needed premises should be replaced by the distinction between committed vs. non-committed (linking) premises. The unexpressed linking premise, while certainly needed, may not be used, but that is not the point for an analyst, the point is that whether an argumentation is committed to a certain linking premise by means of which a particular argument scheme that has been used in the argumentation. The determination of which argument scheme that has been used is made by an analysis of which argumentative indicators that have been used (van Eemeren et al. 2007; Snoeck Henkemans 1996; 2003; 2010).

What then are the implications of all this for the reconstruction of complex argumentation by analogy? Having a dialectical perspective and applying the procedure for finding the unexpressed linking premise will help identifying the constituents for correctly reconstructing a complex of analogical arguments. We saw in section [2.1] that analogical reasoning is not based on mere similarity but on two crucial relations (the vertical determining relation and the horizontal one-to-one relation) operating between the elements in the Analogue and the elements in the Target-Subject. First, in the Analogue, there is a vertical relation between one (or more) element(s) which (in a broad sense) determines its Assigned-Predicate. Secondly, there is a horizontal one-to-one corresponding element* and a one-to-one corresponding Assigned-Predicate* in the TargetSubject. Thus, you can transfer the same determining relation, to hold between the mutatis mutandis one-to-one corresponding element* and the one-to-one corresponding Assigned-Predicate* in the Target-Subject.

This holds for any single argumentation by analogy. Thus, the primary constituents that must be identified are the propositions that express these relations, and the other parts outlined in section [2.1].

In complex argumentation by analogy-if it means a "complex made out of analogical arguments"-there are many analogical arguments which operate in a unified fashion. In a single argumentation by analogy there is one scheme with one element of comparison, and in a complex argumentation by analogy there are a multitude of such arguments, each with one analogical scheme containing one element of resemblance. However, complex argumentation by analogy that is entirely composed of analogical arguments can be complex in three 
ways: either by means of supplying an added element of resemblance for each added single argument, or by means of supplying an additional entire analogue for each added single argument, or by a combination of such single arguments.

In other words, an analogical argumentation can be made complex by adding another whole object of comparison next to the first comparison and not just adding further resemblances to the first comparison. Thus, a complex analogical argumentation can be a complex made out of additional resemblances or of additional objects of comparison. One could easily think that such complex "argumentation by multi-source analogy" would always be a type of "multiple analogical argumentation," in which each single argument is a separate line of defense whereas a complex "argumentation by single-source analogy" amounts to cumulative or complementary coordinatively compound argumentation.

However, that would be an erroneous conclusion, since the pragma-dialectical distinction between multiple and coordinatively compound argumentation is based on whether a single argument by itself is a sufficient defense or not. Nothing per se regulates that each object of comparison is a sufficient analogy with the Target-Subject, nor that each added resemblance by itself is insufficient for the standpoint. A complex "argumentation by single-source analogy" need not necessarily be coordinatively compound, although the usual dialectical reason for pointing out an additional resemblance is that you want to overcome doubts about the analogy between the Analogue and the Target-Subject. It is conceivable that each added resemblance is intended as a separate reason for the analogy between the Analogue and the Target-Subject.

In other words, the pragma-dialectical distinction between multiple and coordinatively compound argumentation cut-across the difference between complex "argumentation by singlesource analogy" and complex "argumentation by multi-source analogy." However, in my judgment most complex argumentation by analogy - whose complexity consists of solely analogical arguments-will have a compound structure, not multiple. In most cases complex argumentation by analogy has one large subordinative part that consists of cumulative coordinatively compound (see section [5]) and would have the following structure:

1. The Target-Subject $\mathrm{TS}_{\mathrm{TS}}$ has the Assigned-Predicate $\mathrm{AP}_{\mathrm{A}}$ *

1.1 The element $\in 1-\in 4$ of the Analogue $_{\mathrm{A}}$ is comparable 
with element $\in 1-\in 4^{*}$ of the Target-Subject $\left.{ }_{\mathrm{TS}}\right)^{46}$

(1.1' The elements $\in 1-\in 4$ of the Analogue $_{\mathbf{A}}$ determines the Analogue $_{\mathrm{A}} \mathrm{s}$ Assigned-Predicate ${ }_{\mathrm{AP}}$ )

1.1.1a The element $\in 1$ of the Analogue A $_{\mathrm{A}}$ correspond one-toone to element $\in 1$ * of the Target-Subject ${ }_{\mathrm{TS}}$

(1.1.1a' The element $\in 1$ of the Analogue $\mathrm{A}_{\mathrm{A}}$ element ${\text { determines the } \text { Analogue }_{\mathrm{A}} \text { 's Assigned-Predicate }}_{\mathrm{AP}}$ )

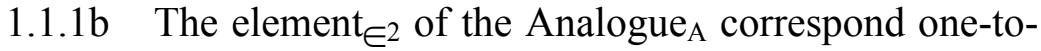
one to element $\in 2$ *of the Target-Subject $\mathrm{TS}_{\mathrm{TS}}$ )

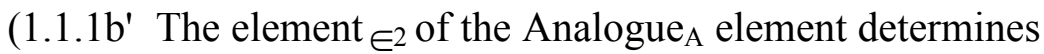
the Analogue $_{\mathrm{A}}$ 's Assigned-Predicate AP $_{\mathrm{P}}$ )

1.1.1c The element $\in_{3}$ of the Analogue A $_{\mathrm{A}}$ correspond one-toone to element $\in 3^{*}$ of the Target-Subject $\mathrm{TS}_{\mathrm{S}}$

(1.1.1c' The element $_{\in 3}$ of the Analogue $_{\mathrm{A}}$ element determines the Analogue $_{\mathrm{A}}$ 's Assigned-Predicate ${ }_{\mathrm{AP}}$ )

1.1.1d The element $\in_{4}$ of the Analogue $_{A}$ correspond one-toone to element $\in 4^{*}$ of the Target-Subject $\mathrm{TS}_{\mathrm{S}}$

(1.1.'1d' The element $\in_{4}$ of the Analogue $_{\mathrm{A}}$ element determines the Analogue $_{\mathrm{A}^{\prime}}$ S Assigned-Predicate AP $_{\mathrm{P}}$ )

The structure can be seen in Figure 3, on the next page.

What does all this mean in actual and concrete cases of complex argumentation by analogy? That question will be addressed in the next section.

\subsection{A concrete example}

In this section I will show how to reconstruct a very complex case of analogical argumentation with my analysis of analogical argument schemes. The example comes from a real legal case, and it has been said to be "as fine a closing argument as has ever been delivered in an American courtroom" (Lief et al. 1998, p. 123). The argumentation was used by attorney Gerry Spence in the case of Silkwood vs. Kerr-McGee Corporation, who argued

${ }^{46} 1.1 \& 1.1$ ' yields that the Analogue and the Target-Subject are similar in the all aspects relevant for the Assigned-Predicate, this can in complex argumentation be simplified as one proposition, see next section in the concrete example.

(C) André Juthe. Informal Logic, Vol. 35, No. 3 (2015), pp. 378-445. 


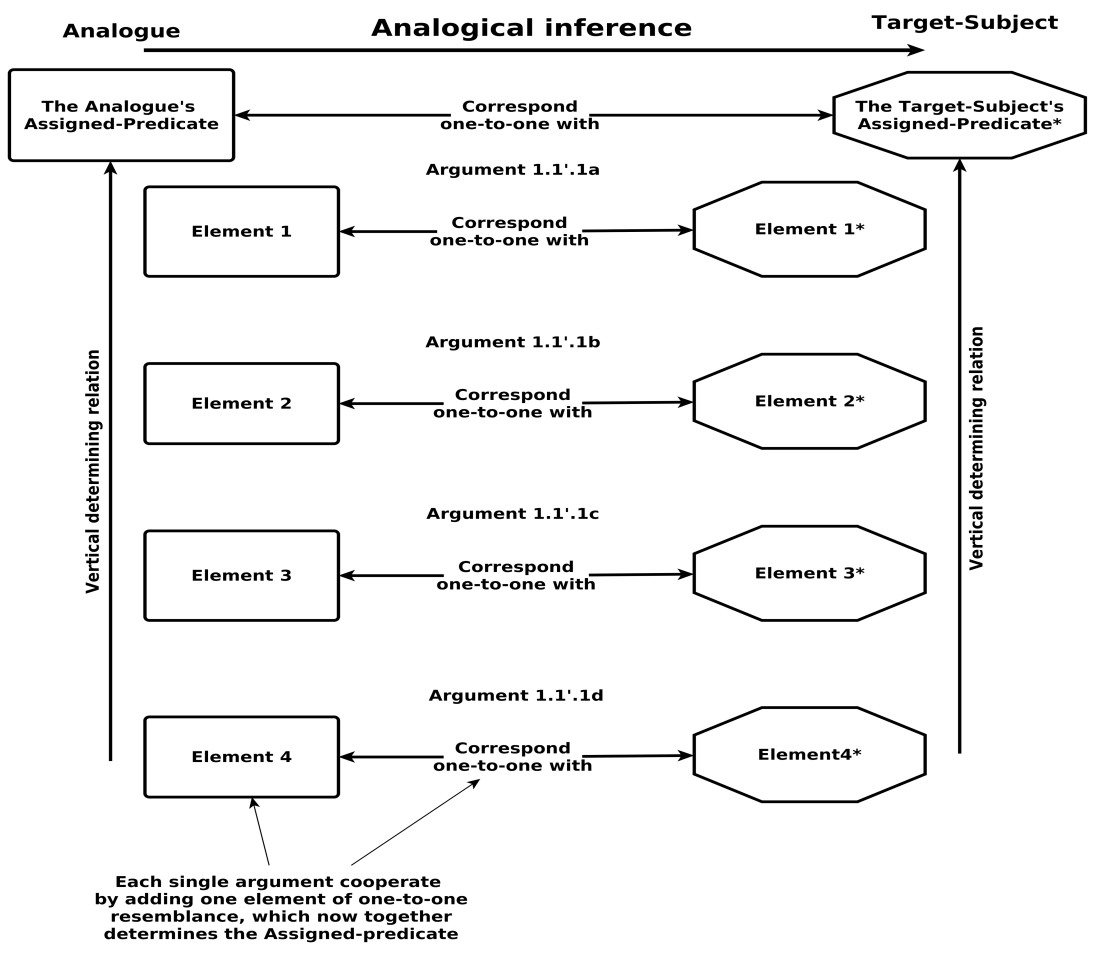

Figure 3. Complex argumentation by analogy

for strict liability. ${ }^{47}$ The technician Karen Silkwood was a young lab analyst working with grinding and polishing plutonium pins used to make fuel rods from nuclear reactors. Her father brought a suit against the company on the behalf of her children ${ }^{48}$ after it was discovered that parts of her body had been exposed to dangerously high levels of plutonium radiation (Lief et al., 1998, 120). Spence's closing argument used the analogy of the escaping lion with great rhetorical effect on the jury:

Well, we talked about "strict liability" at the outset, and you'll hear the court tell you about "strict liability," and it simply means: "If the lion got away, Kerr-McGee has to

\footnotetext{
${ }^{47}$ The purpose of strict liability law is to prevent unnecessary harm. The justification for 'strict liability' is that if anyone is engaged in a dangerous activity, such a person is held to a stricter standard of responsibility for taking care for any damage that might be caused by that activity.

${ }^{48}$ Karen Silkwood died in a mysterious one-car accident on the way to meeting a reporter from the New York Times. Karen was to provide information that safety and quality controls at Kerr-McGee on the making of the fuel rods had been falsified (Walton 2013) pp. 281-282.
}

(C) André Juthe. Informal Logic, Vol. 35, No. 3 (2015), pp. 378-445. 
pay." It's that simple-that's the law. You remember what I told you in the opening statement about strict liability? It came out of the Old English common law. Some guy brought an old lion on his ground, and he put it in a cage - and lions are dangerous - and through no negligence of his own, through no fault of his own, the lion got away. Nobody knew how-like in this case, "nobody knew how." And, the lion went out and he ate up some people - and they sued the man. And they said, you know: "Pay. It was your lion, and he got away." And the man says: "But I did everything in my power-I had a good cage-had a lock on the door-I did everything that I could-I had security-I had trained people watching the lion-and it isn't my fault that he got away." Why should we punish him? They said: "We have to punish him-we have to punish you-you have to pay." You have to pay because it was your lion-unless the person who was hurt let the lion out himself. That's the only defense in this case: unless in this case Karen Silk wood was the one who intentionally took the plutonium out, and "let the lion out," that is the only defense, and that is why we have heard so much about it.... Strict liability: "If the lion gets away, Kerr-McGee has to pay," unless Karen Silkwood let the lion loose. What do we have to prove? Strict liability. Now, can you see what that is? The lion gets away. We have to do that. It's already admitted. It's admitted in the evidence. They admit it was their plutonium. They admit it's in Karen Silkwood's apartment. It got away. And, we have to prove that Karen Silkwood was damaged. That's all we have to prove. Our case has been proved long ago, and I'm not going to labor you with the facts that prove that. It's almost an admitted fact, that it got away, and that she was damaged. (Lief et al. 1998 p. 125, 129.)

Each single argumentation that comprises this complex argumentation employ argument by conclusive different-domains analogies. The complete argumentation structure with all essential aspects specified for an all-inclusive evaluation would be: ${ }^{49}$

49 The question of reconstruction is also a question about which features are necessary to make explicit. Of course you could just reconstruct it into an argumentation with very few premises:

1. The McGee Corp. is strictly liable for the harm the plutonium caused Silkwood.

1.1 The Lion owner was strictly liable for the harm the lion caused.

(C) André Juthe. Informal Logic, Vol. 35, No. 3 (2015), pp. 378-445. 
1. The McGee Corp. is strictly liable for the harm the plutonium caused Silkwood.

1.1 The plutonium got away and damaged Karen Silkwood.

(1.1' These two facts are sufficient to make McGee Corp strictly liable.)

1.1.1 The plutonium was found in Karen Silkwood's apartment.

1.1'.1 The fact that the lion got away and killed people was sufficient to make the lion owner strictly liable for the people the lion killed in the case of the lion owner.

(1.1'.1' The McGee Corp. plutonium case is comparable to all the relevant aspects of strict liability in the case of the lion owner.)

(1.1'.1').1a The McGee Corp. plutonium case is comparable to the case of the lion owner with respect to knowledge of the danger.

$\left(\left(1.1^{\prime} .1^{\prime}\right) .1 a^{\prime}\right.$ Knowledge of the danger was relevant for strict liability in the case of the lion owner.)

$\left(1.1^{\prime} .1^{\prime}\right) .1 \mathrm{~b}$ The McGee Corp. plutonium case is comparable to the case of the lion owner with respect to ownership.

$\left(\left(1.1^{\prime} .1^{\prime}\right) .1 b^{\prime}\right.$ The ownership of the lion was relevant for strict liability in the case of the lion owner. )

(1.1'.1').1c The McGee Corp. plutonium case is comparable to the case of the lion owner with respect to how the danger escaped (it just got away and nobody knew how).

1.1' The case of Silkwood case is analogous with the lion case.

But that would neither specify all features relevant for an all-inclusive evaluation nor capture the dialectical complexity of the argumentation. This does not mean that the above exceedingly long reconstruction should always be made-you do not need to always specify all the features relevant for an all-inclusive evaluation-sometimes it is sufficient to just focus on some parts that you deem most important for you purpose. However, for sake of completeness and in order to reach the goal of this essay, I provide a full reconstruction that specifies every argument that I think is part of the argumentation as a whole.

(C) André Juthe. Informal Logic, Vol. 35, No. 3 (2015), pp. 378-445. 
$\left(\left(1.1^{\prime} .1^{\prime}.\right) 1 c^{\prime}\right.$ How the lion-the danger-escaped, was relevant for strict liability in the case of the lion owner.)

(1.1'.1').1d The McGee Corp. plutonium case is comparable to the case of the lion owner with respect to proper impeccable security.

$\left(\left(1.1^{\prime} .1^{\prime}\right) .1 \mathrm{~d}^{\prime}\right.$ That the lion owner had proper security, and could not be blamed that the lion escaped, was not sufficient to avoid liability in the case of the lion owner.)

(1.1'.1').1d.1a The McGee Corp. plutonium case is comparable to the case of the lion owner with respect to how being engaged in dangerous activity outweighs any consideration of impeccable proper security.

$\left(\left(1.1^{\prime} .1 '\right) .1 d^{\prime} .1 a^{\prime}\right.$ That dangerous activity outweighs any consideration of impeccable proper security was relevant for strict liability in the case of the lion owner.)

(1.1'.1').1d.1b The McGee Corp. plutonium case is comparable to the case of the lion owner with respect to the only way the lion owner could have avoided liability.

$\left(\left(1.1^{\prime} .11^{\prime}\right) .1 \mathrm{~d} .1 \mathrm{~b}\right.$ ' The only way to avoid liability was (of course) relevant for the case of the lion owner.)

$(1.1 ' .1 ') .1 \mathrm{~d}$ '.1c The only way the lion owner could have avoided strict liability was if the persons who got killed had intentionally freed the lion themselves (but that was not the case).

$\left(\left(1.1^{\prime} .11^{\prime}\right) .1 \mathrm{~d}^{\prime} .1 \mathrm{c}^{\prime}\right.$ Nor yet was it the case that Silkwood herself had released the plutonium.)

What this (dialectically) complex argumentation structure reveals fits very well with my theory on analogical argument schemes. (And, again, it is clear that this cannot be reduced to some other scheme). You can see that it has the same structure as that of Lewis's argumentation. It has a one large subordinative part that, in turn, is composed of cumulative coordinatively compound argumentation, which may be broken down to many single argument by analogy that contain one element of comparison. The only contrast is that this argumentation has another subordinative part of cumulative argumentation: (1.1'.1'.)1d.1a - (1.1'.1'.) 1d'.1c. 
If you reflect on it, you recognize that each linking premise, (1.1'1'.)1a', (1.1'.1'.)1b', etc., expresses the vertical relation of the scheme, which "determines" "strictly liable" (the Assigned-Predicate), ${ }^{50}$ whereas each argument, (1.1'.1'.1a, (1.1'.1'.)1b, etc., expresses the horizontal relation of comparison, i.e., the one-to-one correspondence of each of the elements that "determines" the Assigned-Predicate.

Moreover, you can see that each "argument" with its linking premise would constitute a single analogical argumentation perceived on its own. This is expected if the argument structure is composed of single arguments employing an argument scheme that has the inference configuration that was outlined in section [2]. The vertical determining relation in conjunction with the one-to-one correspondence of resemblance is what enables the transference of acceptability from the "arguments" to the standpoint and what makes analogical arguments materially valid in the sense defined in this paper. Given the context the argumentation is pragmatically valid as well.

Thus, the theory of analogical inference configuration outlined in section [2.] can readily fit into the pragma-dialectical theory of argumentation structures and be used to reconstruct complex argumentation by analogy.

The overall dialectical structure is cumulative coordinatively compound, which is another virtue because that allows for degrees in analogical strength, although analogical inference configuration per see does not allow for degrees; either they are analogous or they are not (Juthe 2005). ${ }^{51}$ That is, the microstructure of schemes taken by themselves does not permit degrees (as it should not) ${ }^{52}$ but the macro-structure of dialectical and logical argumentation structure does. This speaks in favor for this account of analogical argumentation for, in general, you expect analogical argumentation to vary in strength. For instance, you may take away, say, argument (1.1'.1'.)1d and

${ }^{50}$ Except (1.1') which is not the linking premise of an analogical argument anyway.

${ }^{51}$ It of course varies in strength with respect to how clearly the elements of the Analogue and of the Target-Subject resemble each other, just like a deductive argument vary in strength with respect how clearly its premises are true.

${ }^{52}$ Since this system permits that it is an open question whether a given single argumentation transfer the acceptability from the "argument" to the standpoint in a sufficient way, a scheme cannot provide a lower degree of entitlement than that of "generic validity." A correct argument scheme will always invariably make the "argument" confer a reason, ceteris paribus, for the standpoint.

(C) André Juthe. Informal Logic, Vol. 35, No. 3 (2015), pp. 378-445. 
while the argumentation as a whole becomes weaker, it still works as an argumentation and is still effective but to a lesser degree. The overall argumentation structure lends a cumulative support for the major linking premise (1.1'1'). The structure can be seen visually in the graph below (Figure 4).

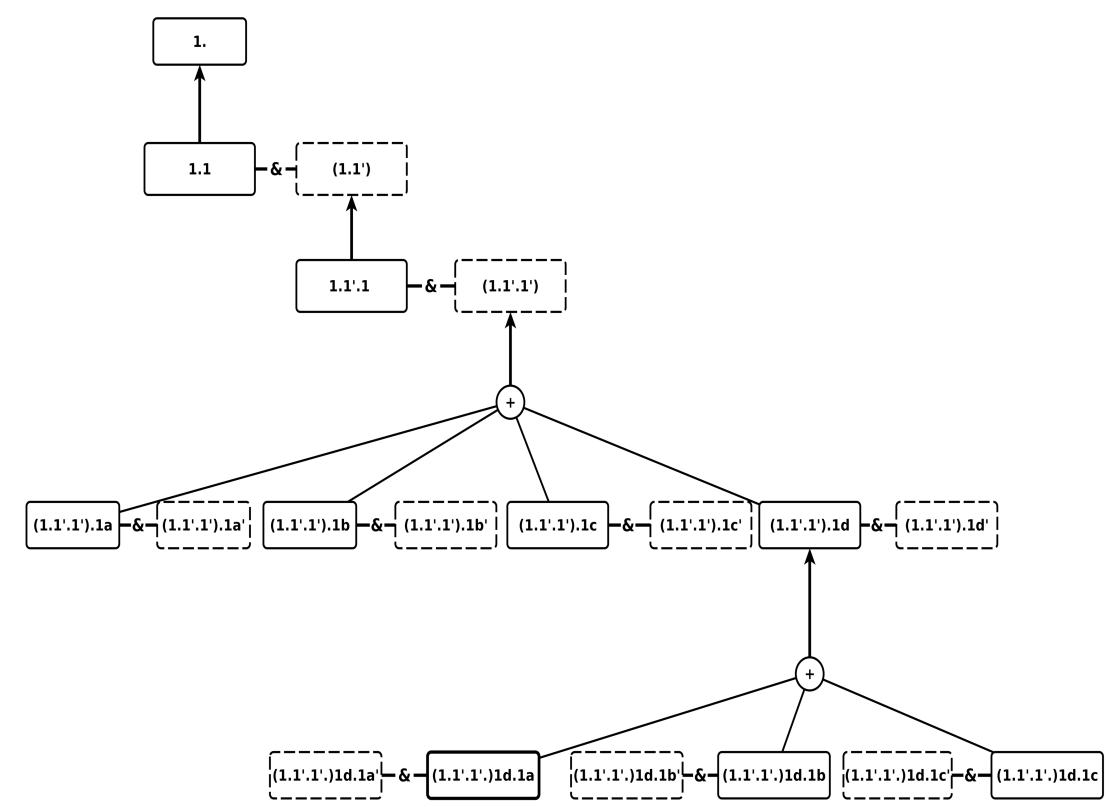

Figure 4. The analogical argumentation of Gerry Spence

I will now very briefly comment on the logical structure. For reasons of space, I will focus only on the logical structure of the overall dialectical structure. As previously noted, a cumulative coordinatively compound argumentation-if you accept Freeman's criterion-will necessarily be logically convergent.

The arguments are all independent in the sense that they do not need each other in order to be relevant for (1.1'.1'), but they are "modally connected" in that each increases the evidence for the major linking premise (1.1'.1'). While the propositions and the inference configuration that constitute a scheme internally are linked, each analogical argument is clearly independently relevant for (1.1'.1'). We find the exact same phenomenon in the reconstructed complex argumentation by Lewis in section [3.5]. It has dialectically speaking an overall cumulative coordinatively compound argumentation that supports the major linking premise and each of these arguments 
converge logically on the same linking premise ${ }^{53}$ and the reason for this uniform pattern brings us to the final point of this paper.

\section{Scheme-stereotypical dialectical argumentation structures}

In this section, I will briefly argue that analogical argument schemes by their very nature promote a certain complex argumentation structure. Even though dialectical argumentation structures are ideally seen as the result of an actual dialogue, they are often the result of a monologue with responses to anticipated critical reactions from the audience of the argumentation. An argument structure should, however, not only be seen as the result of a dialogical exchange to critical reactions, but as a result of an intention to reach a certain goal of convincing the antagonist employing certain argument schemes. Thus, the use of an argumentation that employs a certain argument scheme is connected (as a correlation) to certain argument structures due to the intrinsic inference configuration of the scheme that was used.

This connection between argument structures and argument schemes is reminiscent of a recent development in pragma-dialectical research, focusing on what is called "stereotypical argumentative patterns" (van Eemeren \& Garssen 2014; 2013; van Eemeren 2014). The idea is that contrasting argument schemes of argumentation can be instrumental in reaching the kind of outcome aimed for in certain contexts of communicative activity types. For instance, "causal" argumentation might contribute to establishing the truth of a scientific claim in an academic communicative activity type, whereas arguments that employ analogical schemes may be more useful in legal activity type, and so forth (ibid.). Generally, arguments by analogy are typically embedded in argumentative patterns in two ways: they may be employed to defend another argumentation or they may be defended themselves by another type of argumentation (Eemeren \& Garssen 2014). For instance, an argumentative pattern may consist of pragmatic argumentation defended by an analogical argument in a subordinative argument structure (ibid.).

\footnotetext{
${ }^{53}$ You can see that the pattern in Lewis striptease analogy is exactly the same, just with less complexity. In section [3.5] you can see that argument $1.1^{\prime} .1 \mathrm{a}$ and $1.1^{\prime} .1 \mathrm{~b}$ dialectically lend cumulative support to the major linking premise $1.1^{\prime}$ which enables 1.1. to transfer acceptability to 1 .
}

(C) André Juthe. Informal Logic, Vol. 35, No. 3 (2015), pp. 378-445. 
My idea is, however, not about how regular patterns of standpoints, argument schemes and argument structures are activated to satisfy the institutional conventions of a certain communication activity type. It is not that certain contexts may activate certain argumentative patterns. The idea is rather that the very nature of the inference configuration of the schemes themselves activates certain argument structures. Each type scheme will by itself increase the likelihood of certain argument structures and sometime even make it quite certain. Why does this special pattern of (dialectical) complex argumentation that we saw with the lion analogy and Lewis striptease analogy arise with analogical argumentation? It follows from the protagonist's anticipated response to the critical questions that are germane to the specific scheme he is employing. The standard scheme: ${ }^{54}$

$$
\begin{array}{lll} 
& 1 . & \mathrm{Y} \text { is true of } \mathrm{X}, \text { [Standpoint] } \\
\text { because: } & 1.1 & \mathrm{Y} \text { is true } \mathrm{Z}, \text { [Argument] } \\
\text { and: } & 1.1^{\prime} \quad \mathrm{Z} \text { is comparable to } \mathrm{X} . \text { [Linking premise] }
\end{array}
$$

has the following standard critical questions as testing procedure (van Eemeren et al 2007, pp. 139):

(1) Are the things that are compared actually comparable?

(2) Are there enough relevant similarities between the things that are compared?

(3) Are there any relevant differences between the things that are compared $?^{55}$

These critical reactions pertain to the type of inference that is intrinsic in this scheme, hence the defense will focus on the claim that the Target-Subject and Analogue are comparable. Some of the critical reactions to contrasting types of argumentations will be the same, i.e., they question the acceptability of the "argument," etc. However, the critical question against the inference will, by the nature of the case, be in accordance with the type of argument scheme that is used. Consequently, the defense will tend to give rise to a particular dialectical argumentation structure due to this fact. It is a plain

\footnotetext{
${ }^{54}$ The modified scheme that was outlined in section [2] is no different with respect to a defense against the critical questions, and how a complex argumentation results from a defense against the critical questions that pertains to the analogical scheme.

${ }^{55}$ In a forthcoming paper, "Defense Against Argument by Analogy," I will address more fully the testing procedures of analogical argumentation (Juthe forthcoming (a).
}

(C) André Juthe. Informal Logic, Vol. 35, No. 3 (2015), pp. 378-445. 
empirical and social fact that most critical reactions against argumentation by analogy are directed against the claim of relevant similarities, regardless of context. ${ }^{56}$

The critical issue is whether the analogical claim has sufficient support. Thus, the critical reaction against an analogical argumentation is mostly directed against the comparison that is employed, which means that argumentation that is complex and compound and employs analogical argument schemes will mostly result in a dialectical argumentation structure that is cumulative coordinatively compound with a logical structure that is convergent (since all coordinatively compound is logically convergent). ${ }^{57}$

You could of course have a complementary coordinative compound argument structure, but then the refutation of the counter-argument must be done by means of an analogical argument (if it is supposed to be a complex composed of solely analogical arguments). The same holds for multiple argumenttation or a subordinative argument in a subordinatively compound argument structure.

Thus, (dialectically) complex argumentation that employs analogical argument schemes will mostly be composed of two main argumentation parts, a subordinate part that, in turn, consists of cumulative coordinative compound argumentation. Each analogical argument that builds the complex will have the structure that is common to all singular argumentation by analogy outlined in section [2]. The standpoint will contain the Target-Subject, the argument will contain the Analogue, and both of them will contain the Assigned-Predicate, which is transferred from the argument to the standpoint in virtue of the vertical and horizontal relation expressed by the "argument" and the linking premise. In complex arguments by analogy, the added arguments will cooperate in a cumulative coordinatively compound way in order to lend sufficient support to the claim of analogy.

\footnotetext{
${ }^{56}$ That is my experience. The explanation for this phenomenon follows from the intrinsic nature of analogical inference. In such inference you start with what is accepted and goes to what is not accepted. Thus the starting point is not what is controversial in analogical argumentation and will in most cases not be questioned. The critical responses will instead focus on the inferencelink, which is why this particular argument structure arises.

${ }^{57}$ That is, if you accept Freeman's criterion for logically linked vs logically convergent argumentation structure.
}

(C) André Juthe. Informal Logic, Vol. 35, No. 3 (2015), pp. 378-445. 


\section{Summary and conclusion}

I have, in this paper, tried to show how a theory of analogical inference fits the pragma-dialectical system of argument schemes and argument structures. I have also tried to provide a unified account of argument schemes and of arguments that has formal validity. Argumentation by analogy employs a sui generis type of inference that operates via two critical relations, the vertical and the horizontal relation. Arguments by analogy operate via material inferences and are materially "valid" but are not formally valid.

Argumentation by analogy is not unique in that respect since all argument schemes in the pragma-dialectical typology are "substantial schemes" whose inference configurations operate by a substantial principle and not a formal principle. Each scheme has its own intrinsic standard of validity"scheme-specific-validity" - and formal validity is only one type of validity. Every argument scheme is a species of a generic validity and this yields a unified account of argument schemes, validity and inference. Pragmatic validity is a notion related to the rules of the critical discussion and the success of the argumentation in the discussion, whereas formal and material validity are related to the argumentation perceived in isolation from any dialectical situation. Logical argument structure must be distinguished from dialectical argument structure because they are made from different disciplinary perspectives. However, both are necessary for an adequate dealing with argumentation.

The reconstruction of complex argumentation by analogy refers here to argumentative complexity that consists of several analogical argument schemes. In such structure each argument provides one element of comparison in order to lend support to the standpoint (or another argument or linking premise). However, you could also have a complex analogical argument structure in which each single argument employs an entire additional object of comparison instead of single arguments that just add an element of resemblance, or a structure which utilizes a combination of such arguments. However, this difference has no bearing as to whether such argument structure is coordinatively compound or multiple.

If you correctly reconstruct (dialectically) complex analogical argumentation in accordance with the suggested procedure for finding the unexpressed premise, you will often find a certain pattern. Complex argumentation by analogy tends 
to result in a certain argument structure due to the intrinsic nature of the scheme itself and the critical questions that pertains to that scheme. This should not be confused with "stereotypical argumentative patterns" which means that certain argument schemes and argument structures are activated to satisfy the institutional conventions of certain communication activity types.

\section{References}

Bermejo-Luque, Lilian. (2014). "Deduction without dogmas: the case of moral analogical argumentation," Informal Logic, Vol. 34, No.3 (2014), pp. 311-336.

Brandom, R.B. (1994). Making it explicit: Reasoning, representing, and discursive commitment. Cambridge, MA: Harvard University Press. (This reference is from Brigandt (2010)).

Brigandt, Ingo. (2010). "Scientific Reasoning Is Material Inference: Combining Confirmation, Discovery, and Explanation," International Studies in the Philosophy of Science 24(1): 31-43.

Burbidge, John. (1990). Within Reason-A Guide to Nondeductive Reasoning. Peterborough, ON: Broadview Press.

van Eemeren, Frans H. (2010). Strategic Maneuvering in Argumentative Discourse. Extending the Pragma-dialectical Theory of Argumentation. Amsterdam-Philadelphia: John Benjamins.

van Eemeren, Frans H. (2014) "Analyzing Contextualized Argumentative Discourse: Strengthening the Relationship between Rigotti's Aspirations and Pragma-Dialectics" in: Gobber, Giovanni and Rocci, Andrea (eds.) Language, reason and education: Studies in honor of Eddo Rigotti, Series: Sciences pour la communication, Volume 113.

van Eemeren, F.H. \& Grootendorst, R. (1982). "The speech acts of arguing and convincing in externalized discussions," Journal of Pragmatics 6: 1-24.

van Eemeren, F.H., \& Grootendorst, R. (1984). Speech Acts in Argumentative Discussions: A Theoretical Model for the Analysis of Discussions Directed towards Solving Conflicts of Opinion. Dordrecht: Floris Publications.

van Eemeren, Frans H. \& Grootendorst, R. (2004). A Systematic Theory of Argumentation: The Pragma-dialectical Approach, Cambridge: Cambridge University Press. 
Van Eemeren, Frans H., Grootendorst, Rob \& Snoeck Henkemans, A. Francisca. (2002). Argumentation. Analysis, Evaluation, Presentation, Mahwah, NJ: Lawrence Erlbaum Associates.

van Eemeren, Frans H., Houtlosser, Peter \& Snoeck Henkemans, A. Francisca. (2007). Argumentative Indicators in Discourse: a Pragma-dialectical Study. (Argumentation library, 12). Dordrecht: Springer.

van Eemeren, Frans H. \& Garssen, Bart. (2013). "Argumentative patterns in discourse." in D. Mohammed \& $\mathrm{M}$. Lewiński, (Eds.), Virtues of Argumentation: Proceedings of the 10th International Conference of the Ontario Society for the Study of Argumentation (OSSA), (pp. 1-15). Windsor, ON: OSSA.

van Eemeren, Frans H. \& Garssen, Bart. (2014). "Argumentation by Analogy in Argumentative Stereotypical Patterns," in Henrique, Jales Ribeiro (Ed.), Systematic Approaches To Argument By Analogy (pp. 41-56). (Argumentation Library vol. 25) Springer: Dordrecht.

Ennis, R.H. (1982) "Identifying Implicit Assumptions." Synthese 51: 61-68.

Garssen, Bart. (2001). "Argument Schemes," in Frans van Eemeren (Ed.), Crucial Concepts in Argumentation Theory. Amsterdam: Amsterdam University Press.

Gerritsen, Susanne. (2001). "Unexpressed Premises," in Eemeren, F.H. van (Ed.), Crucial concepts in argumentation theory (pp. 51-80). Amsterdam: Sic Sat.

Godden, David, M. (2005). "Deductivism as an Interpretive Strategy: A Reply to Groarke's Recent Defense of Reconstructive Deductivism," Argumentation and Advocacy 41: 168-183.

Gough, James \& Tindale, Christopher. (1985). “"Hidden' or 'missing' premises," Informal Logic 7(2\&3): 100-106.

Govier, Trudy. (1972). "Presuppositions, Conditions, and Consequences," Canadian Journal of Philosophy 1(4): 443456.

Govier, Trudy. (1989). "Analogies and Missing Premises." Informal Logic 2(3): 141-152.

Guarini, Marcello. (2004). "A Defense of Non-deductive Reconstructions of Analogical Arguments," Informal Logic, 24(2): 153-168. 
Hitchcock, D.L., \& Wagemans, J.H.M. (2011). "The pragmadialectical account of argument schemes," in E.T. Feteris, B.J. Garssen \& A.F. Snoeck Henkemans (Eds.), Keeping in touch with pragma-dialectics (pp. 185-205). Amsterdam: Benjamins.

Hitchcock, David. (2015). "Freeman's Syntactic Criterion for Linkage," Informal Logic, 35(1): 1-31.

Hitchcock, David. (1998). "Does the Traditional Treatment of Enthymemes Rest on a Mistake?" Argumentation 12: 15-37.

Holtzman, Seth. (1997). "A Philosophical Re-examination of Presupposition." Unpublished ms. Present position: Catawba College, Salisbury, NC.

Juthe, André (2005). "Argument by Analogy," Argumentation 19: 1-27.

Juthe, André (2009) "Refutation by Parallel Argument," Argumentation 23: 133-169.

Juthe, André. (2014). "A Systematic Review of Classifications of Arguments by Analogy," in Henrique Jales Ribeiro (Ed.), Systematic Approaches to Argument by Analogy (pp. 109127). Springer: Dordrecht.

Juthe, André. (forthcoming a). Defense Against Arguments by Analogy.

Juthe, André. (forthcoming b). Argumentation by Analogy, A Systematic and Analytical Study of an Argument Scheme.

Lief, H. Michael S. \& Caldwell, Mitchell Bycel, Benjamin. (1998). Ladies and Gentlemen of the Jury: Greatest Closing Arguments in Modern Law. New York: Scribner.

Macagno, Fabrizio \& Walton, Douglas. (2006). Argumentative Reasoning Patterns, ECAI 2006, Riva del Garda, August 28 September 2, 2006.

(Available at SSRN: http://ssrn.com/abstract=1751683 or http://dx.doi.org/10.2139/ssrn.1751683).

Massey, Gerald J. (1975). "Are there any Good Arguments that Bad Arguments are Bad?" Philosophy in Context (4): 61-77.

McGee, V. (1985). "A counterexample to Modus Ponens,, The Journal of Philosophy 82(9): 462-471.

Pinto, Robert C. (2006) "Evaluating Inferences: The Nature and Role of Warrants," Informal Logic 26(3): 287-317.

Plumer, Gilbert. (1999). "Necessary Assumptions," Informal Logic 19(1): 41-61.

Russell, Bertrand. [1913] (2013). The Problems of Philosophy, Project Gutenberg's [Electronic]. Accessible: $<$ http://www.gutenberg.org/ebooks/5827> [2015-03-10]. 
Shecaira, Fábio P. (2013). "Analogical arguments in Ethics and Law: A Defence of a Deductivist Analysis," Informal Logic, 33(3): 406-437.

Smith, J.W. (1986). Reason, Science and Paradox. New Hampshire: Croom Helm Ltd., Provident House.

Smith, J.W. (1988). Essays on ultimate questions: Critical discussions of the limits of contemporary philosophical inquiry. (Avebury Series in Philosophy). Aldershot.

Snoeck Henkemans, A. Francisca. (1997). Analysing Complex Argumentation. The Reconstruction of Multiple and Coordinatively Compound Argumentation in a Critical Discussion, $2^{\text {nd }}$ ed. Amsterdam: Sic Sat.

Snoeck Henkemans, A. Francisca. (2000). "State-of-the-Art: The Structure Of Argumentation," Argumentation 14: 447473.

Snoeck Henkemans, A. Francisca. (2014). "Speech act theory and the study of argumentation." Studies in Logic, Grammar and Rhetoric 36 (49): 41-58.

Snoeck Henkemans, Francisca. (2003). "Indicators of Complex Argumentation," in J. A. Blair, et al. (eds.), Informal Logic at 25: Proceedings of the Windsor Conference, (pp. 1-9). CDROM, Windsor, ON: OSSA.

Snoeck Henkemans, A. Francisca. (2010). “Anyway' and 'even' as indicators of complex argumentation." Cogency, 2(1), 81-94.

Snoeck Henkemans, A. Francisca. (1996). "Indicators of independent and interdependent arguments: 'anyway' and 'even'," in Johan van Benthem, Frans H. van Eemeren, Rob Grootendorst \& Frank Veltman (Eds.), Logic and Argumentation (pp. 77-87). Amsterdam: Koninklijke Nederlandse Akademie van Wetenschappen, Taylor and Francis Group.

Steinhart, E. C. (2001). The Logic of Metaphor, Analogous

Parts of Possible Worlds. Dordrecht: Kluwer Academic Publishers.

Theron, S. (1997) "Argument forms and Argument from Analogy", Acta Philosophica 6(2): 303-326.

Tomic, Taeda. (2013). "False Dilemma: A Systematic Exposition," Argumentation 27: 347-368.

Tropman, Elizabeth. (2010). "Intuitionism and the SecondaryQuality Analogy in Ethics," Journal of Value Inquiry 44: 3145.

Walton, Douglas N. (2001). "Abductive, Presumptive and Plausible Arguments," Informal Logic 21(2): 141-169. 
Walton, Douglas. (2013). "Argument from Analogy in Legal Rhetoric," Artificial Intelligence \& Law 21(3): 1-22.

Walton, Douglas N. \& Reed, Chris A. (2002). "Argumentation Schemes and Defeasible Inferences," in Guiseppe Carenini, Floriana Grasso \& Chris Reed (Eds.), Workshop on Computational Models of Natural Argument. ECAI 2002, 15th European Conference on AI, 2002.

Walton, Douglas, Reed, Chris and Macagno, Fabrizio. (2008). Argumentation Scheme. Cambridge: Cambridge University Press.

Weitzenfeld, J. S. (1984). "Valid Reasoning by Analogy,"

Philosophy of Science 51: 137-149. 